\title{
Center-point steering analysis of tracked omni-vehicles based on skid conditions
}

\author{
Yuan Fang ${ }^{1}$, Yunan Zhang ${ }^{1}$, Yinghui Shang ${ }^{1}$, Tao Huang ${ }^{2}$, and Mengfei Yan ${ }^{1}$ \\ ${ }^{1}$ Department of Arms and Control Engineering, Army Academy of Armored Forces, Beijing, 100072, China \\ ${ }^{2}$ Unit 63983 of People's Liberation Army (PLA), Wuxi, 214035, China \\ Correspondence: Yuan Fang (fangyuan_1124@foxmail.com) and Yunan Zhang (zhang_yunan@ sina.com) \\ Received: 11 January 2021 - Revised: 3 April 2021 - Accepted: 6 April 2021 - Published: 18 May 2021
}

\begin{abstract}
Existing center-point steering models of a tracked omni-vehicle seldom consider the skid of the track (roller) grounding section, which is inconsistent with the actual steering process. In this study, for the three typical layout types, rectangular, hybrid, and centripetal, the steady center-point steering motion of a tracked omni-vehicle under skid conditions is analyzed and a correction model is investigated. The numerical solution of the absolute lateral offset of the steering pole is obtained, and the influences of various structural parameters on the numerical solution are discussed. The steering angular velocity reduction coefficient is calculated, and the angular velocity of vehicles is corrected. The simulation of center-point steering motion is carried out on eight virtual prototypes, and the center-point steering motion experiment is carried out on three physical prototypes. The results show that the established correction model is more in line with the steering reality of the tracked omni-vehicle, and it can play a role in correcting the center-point steering angular velocity.
\end{abstract}

\section{Introduction}

An omnidirectional mobile robot (vehicle) system can move in any direction for the 3 degrees of freedom on a plane, and it can also rotate at any angle with zero radius in situ, with flexible movement and convenient control. It has unparalleled advantages in narrow areas requiring high speed and high mobility (Wang, 2018). With the exception of robots containing running mechanisms similar to universal wheels (caster wheels) (Cao, 2018; Clavien et al., 2018; Yang et al., 2018) and robots in the form of a single ball (Karavaev and Kilin, 2017; Madhushani et al., 2017), omnidirectional mobile robots are divided into wheeled and tracked omni-robots according to different running mechanisms.

The running mechanisms that constitute wheeled omnirobots (hereinafter referred to as the "wheeled omnimechanisms") mainly include the Mecanum wheel (Wang, 2018), alternate wheel (Park et al., 2016), MY wheel (Tong, 2017), and spherical omnidirectional wheel (Tadakuma et al., 2008, 2009). Poor load capacity, high requirements for flatness and cleanliness of the walking environment, and poor off-road and obstacle crossing performance are the common shortcomings of wheeled omni-robots. One of the improvement measures is to develop tracked omni-robots.

The running mechanisms of tracked omni-robots (hereinafter referred to as the "tracked omni-mechanisms") mainly include the free-roller track (Chen et al., 2002), Vuton crawler (Roh et al., 2013), Mecanum wheel crawler (Mortensen Ernits et al., 2017; Guan and Yuan, 2019), omnidirectional mobile track (Zhang, 2017; Li et al., 2012; Xing et al., 2018; Ma et al., 2015), planar omnidirectional crawler mobile mechanism (Tadakuma et al., 2017; Takane et al., 2019; Yu et al., 2020), omni-crawler (Tadakuma et al., 2012, 2014), and other omnidirectional tracks. According to whether it has the ability of active movement in two noncollinear directions, the tracked omni-mechanism can be divided into two types: active tracked and semi-active tracked omni-mechanisms. An active tracked omni-mechanism has problems such as a complex structure and poor load capacity. The semi-active tracked omni-mechanism has strong load capacity and a wide range of applications, and it is suitable for small robots (Roh et al., 2013; Zhang and Yang, 2018) and large machinery (Mortensen Ernits et al., 2017; Fang et al., 2020). From the perspective of the projection of the 
grounding section of the running mechanism, an omnidirectional mobile track is the more representative of a semi-active tracked omni-mechanism. The research on vehicles using the omnidirectional mobile track (hereinafter referred to as a "tracked omni-vehicles") is more mature (Zhang and Yang, 2018; Zhang and Huang, 2015).

The center-point steering motion is one of the important motion forms of a tracked omni-robot. The center-point steering theory of a traditional tracked vehicle (such as the tank) cannot be directly applied to a tracked omni-robot. For a tracked omni-vehicle with a rectangular layout, Huang et al. (2014) analyzed the velocity relationship between the base point on the track grounding section and the maximum slip point during the steering process and obtained the relationship between the maximum steering slip ratio and the structural parameters of the vehicle. Huang et al. (2014) lacked analysis of other points on the track except the maximum slip point. The maximum steering slip ratio cannot represent all the characteristics of the center-point steering motion of the vehicle. For a tracked omni-vehicle with a rectangular layout, Zhang et al. (2015) analyzed the velocity relationship between the center point of the grounding section of a single track and other points during the turning process and obtained the expression of the average slip velocity of the grounding section of a single track. Zhang et al. (2015) lacked an analysis of the track layout and did not consider the impact of the track slip on the steering angular velocity of the vehicle. For a tracked omni-vehicle with hybrid layout, Yang et al. (2019b) analyzed the influence of the roller offset angle on steering motion from the perspective of steering torque. Yang et al. (2019b) lacked design parameter analysis other than the roller offset angle, and lacked the analysis of the center-point steering motion of the whole vehicle.

A theoretical analysis of center-point steering motion should be carried out for a variety of typical layout tracked omni-vehicle, and a correction model should be established. The research object should be all the points of the track grounding section while focusing on the analysis of the motion relationship between the track grounding section and the vehicle. The correction model can correct the angular velocity and time of the vehicle, as well as give the relationship between the design parameters such as the roller offset angle and the steering performance. This allows the designer to evaluate the steering performance of the vehicle during the design stage and then optimize the design.

The current design process of the tracked omni-vehicle, as shown in Fig. 1, is divided into three steps.

1. Select or design the preliminarily structure and layout of the mobile mechanism according to the design requirements.

2. Establish a motion model of the vehicle. Because the slip in translational motion can be ignored, the performance and anisotropy of translational motion are analyzed based on the ideal motion model. Because the slip in the center-point steering motion cannot be ignored, the performance of the center-point steering motion cannot be analyzed based on the ideal motion model, and the performance can only be analyzed based on experience. Based on the results of the analysis, the performance of the vehicle's translational and center-point steering motion is evaluated. Through the evaluation results, the optimal value of the roller offset angle (generally $45^{\circ}$ ) is obtained.

3. Compare the theoretical results with the design requirements to see if the design requirements are met.

Analyzing the performance of the center-point steering motion of the vehicle based on experience introduces uncertainty. The performance of the center-point steering motion of the vehicle should be analyzed according to the content in the dashed box in Fig. 1. If the layout of the vehicle to be designed belongs to one of the three types studied in this paper, then the theoretical model given in this article will be directly applied. If not, we should first analyze the velocity and force of the track grounding section and then obtain the correction model of the vehicle. According to the correction model, the numerical solution of absolute lateral offset distance of the steering pole is obtained. The center-point steering motion performance of the vehicle is evaluated according to the numerical solution.

Based on an omnidirectional mobile track, in this study, the center-point steering motion of the three typical types of tracked omni-vehicles, with rectangular, hybrid, and centripetal layouts, is analyzed and the mathematical model is established. Eight virtual prototypes are established in ADAMS, and the center-point steering motion simulation is performed. Three physical prototypes are established, and center-point steering experiments are performed. The simulation and experimental data are analyzed and compared with the theoretical model. The research results of this study can provide guidance for the structural design of the semi-active tracked omni-mechanism represented by the omnidirectional mobile track and a vehicle with this mechanism, and the results can provide a basis for analysis and evaluation. The research results of this study can also provide a reference for the research of an active tracked omni-mechanism and a vehicle with this mechanism and other tracked omni-robots.

\section{Typical layout and ideal kinematics equation}

The typical layout types of tracked omni-vehicles and other tracked omni-robots are mainly rectangular (Mortensen Ernits et al., 2017; Zhang and Yang, 2018; Zhang and Huang, 2015; Zhang et al., 2015; Huang et al., 2014; Singh et al., 2017; Hua and Zhang, 2019; denoted Type I), hybrid (Yang et al., 2019a, b; Liu et al., 2018; denoted Type II), and centripetal layouts (Chen et al., 2002; Roh et al., 2013; Y. Zhang et al., 2017, 2019; Zhang, 2018; X. Zhang et al., 2016; denoted Type III). A schematic of the layout of the three types 


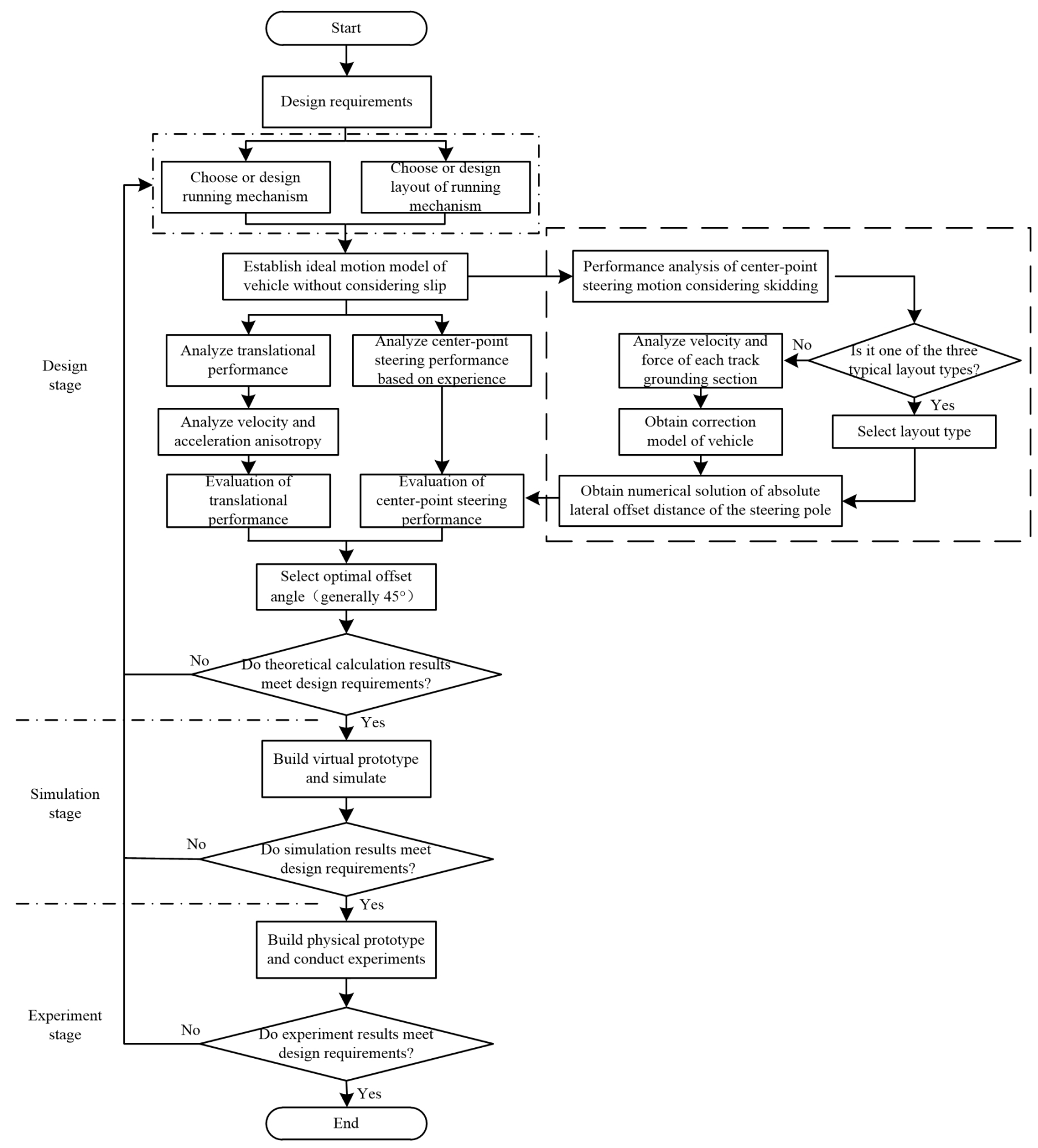

Figure 1. Design process of tracked omni-vehicle.

of tracked omni-vehicles is shown in Table 1, where $p$ represents the types, with $p=1,2,3$ (refer to Appendix A for the meaning of all of the symbols in this article). The tracks are numbered in Table 1. The solid rectangle represents the geometric projection of the track grounding section, the short oblique line represents the axis of the ground roller, and the dotted line represents the vehicle body. The Type III vehicle with two tracks can complete the center-point steering motion but cannot complete the omnidirectional motion, so it is not an omni-vehicle and it is distinguished by brackets in Table 1. 
Table 1. Typical layout of tracked omni-vehicles.

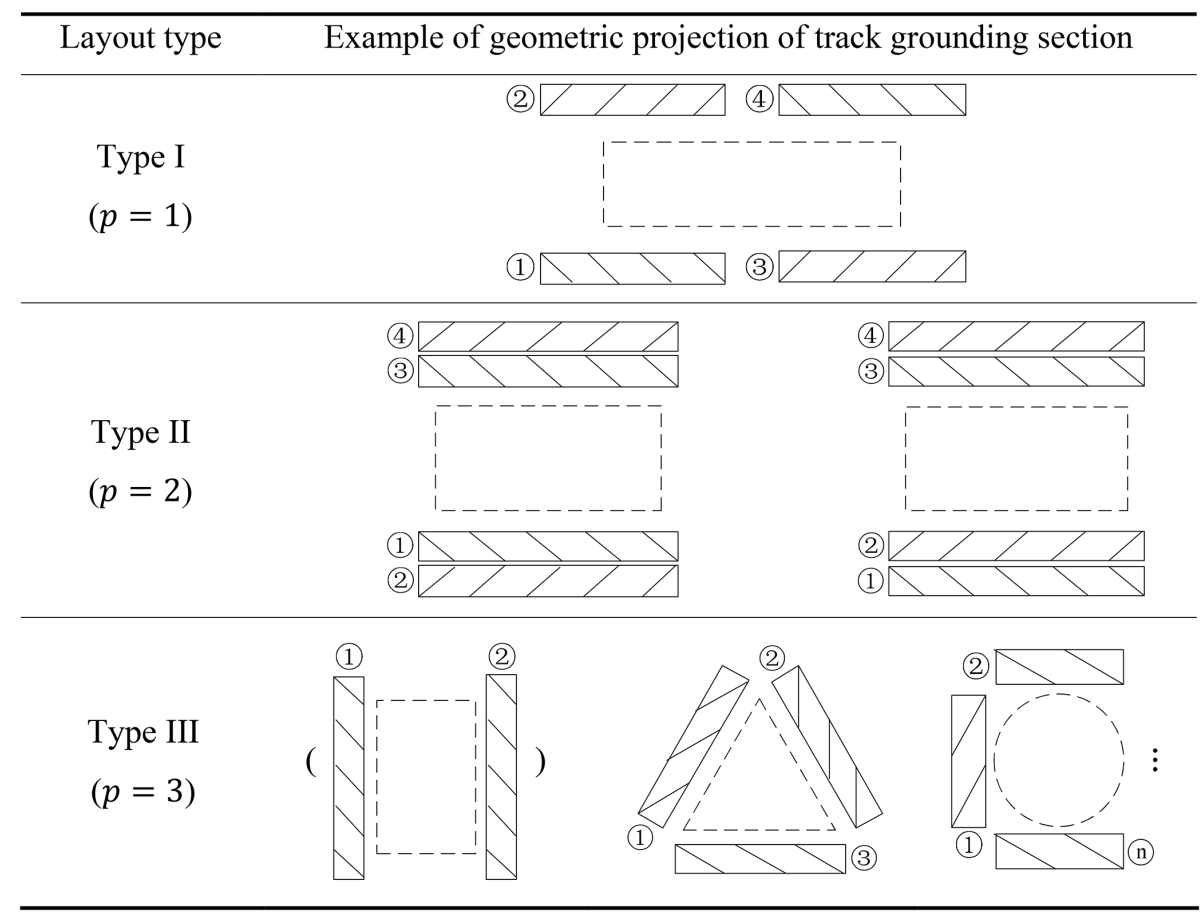

According to the ideal inverse kinematics equation of the tracked omni-vehicle given in Zhang and Huang (2015) without considering the slip, the ideal inverse kinematics equations of the three types shown in Table 1 can be obtained as shown in Eq. (1), wherein the subscript $i$ represents the number $i$ track of the vehicle, $i=1,2,3, \ldots n$. The applicable condition of Eq. (1) is that the track has a shorter landing length (Zhang and Huang, 2015):

$$
\begin{aligned}
& \left(\begin{array}{llllll}
\omega_{p 1} & \omega_{p 2} & \cdots & \omega_{\mathrm{pi}} & \cdots & \omega_{p n}
\end{array}\right)^{T} \\
& m=\mathbf{J}_{\omega p}\left(\begin{array}{llll}
v_{y p} & v_{x p} & \omega_{z p}
\end{array}\right)^{T},
\end{aligned}
$$

where

$$
\mathbf{J}_{\omega p}=\left(\begin{array}{ccc}
\frac{\sin \eta_{p 1}}{r_{p 1} \sin \alpha_{p 1}} & \frac{\cos \eta_{p 1}}{r_{p 1} \sin \alpha_{p 1}} & \frac{l_{p 1} \sin \left(\eta_{p 1}-\beta_{p 1}\right)}{r_{p 1} \sin \alpha_{p 1}} \\
\frac{\sin \eta_{p 2}}{r_{p 2} \sin \alpha_{p 2}} & \frac{\cos \eta_{p 2}}{r_{p 2} \sin \alpha_{p 2}} & \frac{l_{p 2} \sin \left(\eta_{p 2}-\beta_{p 2}\right)}{r_{p 2} \sin \alpha_{p 2}} \\
\vdots & \vdots & \vdots \\
\frac{\sin \eta_{\mathrm{pi}}}{r_{\mathrm{pi}} \sin \alpha_{\mathrm{pi}}} & \frac{\cos \eta_{\mathrm{pi}}}{r_{\mathrm{pi}} \sin \alpha_{\mathrm{pi}}} & \frac{l_{\mathrm{pi}} \sin \left(\eta_{\mathrm{pi}}-\beta_{\mathrm{pi}}\right)}{r_{\mathrm{pi}} \sin \alpha_{\mathrm{pi}}} \\
\vdots & \vdots & \vdots \\
\frac{\sin \eta_{p n}}{r_{p n} \sin \alpha_{p n}} & \frac{\cos \eta_{p n}}{r_{p n} \sin \alpha_{p n}} & \frac{l_{p n} \sin \left(\eta_{p n}-\beta_{p n}\right)}{r_{p n} \sin \alpha_{p n}}
\end{array}\right)^{T} .
$$

In the formula, $\eta_{\mathrm{pi}}=\theta_{\mathrm{pi}}+\alpha_{\mathrm{pi}}, v_{x p}$ is the translational velocity along the $x$ axis and $v_{y p}$ is the translational velocity along the $y$ axis. $\omega_{z p}$ is the angular velocity of the theoretical center-point steering motion without considering slippage (hereinafter referred to as "ideal center-point steering motion"). $\omega_{\mathrm{pi}}$ is the speed of the driving sprocket, $r_{\mathrm{pi}}$ the radius of the pitch circle of the driving sprocket, and $\alpha_{\mathrm{pi}}$ the offset angle of the roller, $\alpha_{\mathrm{pi}} \in\left(-\frac{\pi}{2}, \frac{\pi}{2}\right) / . \beta_{\mathrm{pi}}$ is the angle between the center of the track and the center of the vehicle, and $l_{\mathrm{pi}}$ is the distance between the center of the track and center of the vehicle. $\theta_{\mathrm{pi}}$ is the angle between the coordinate system of the track and the coordinate system of the vehicle. The necessary condition for the vehicle to achieve omnidirectional motion is that the velocity inverse Jacobian matrix of the system is full rank (Wang, 2018), i.e., $\operatorname{rank}\left(\mathbf{J}_{\omega p}\right)=3$. It should be pointed out that Eq. (1) is also the ideal inverse kinematics equation of the omni-platform composed of Mecanum wheels. This means that the essence of Eq. (1) is to equate the omnidirectional mobile track to the Mecanum wheel located in the center of the track, that is, ignoring all grounding points except the center point of the track.

Letting $L_{\text {pi }}$ be the length of the track grounding section, generally, the radius of each driving sprocket of the same vehicle is the same and the grounding length of each track is the same, i.e., $r_{\mathrm{pi}}=r_{p 1}$ and $L_{\mathrm{pi}}=L_{p 1}$. If only the centerpoint steering motion is studied, the rotation speed of all driving sprockets in a vehicle can be set to be the same, i.e., $\omega_{\mathrm{pi}}=\omega_{p 1}$.

The Type I vehicle consists of four tracks, which are located at the four corners of the vehicle body. Tracks (1) and (2) are symmetrical along the longitudinal axis of the vehicle body, tracks (3) and (4) are symmetrical along the longitudinal axis of the vehicle body, tracks (1) and (3) are symmetrical along the transverse axis of the vehicle body, and tracks (2) and (4) are symmetrical along the transverse axis 
of the vehicle body. Tracks (1) and (4) have the same roller offset angle, and tracks (2) and (3) have the same roller offset angle. The signs of the roller offset angles of tracks (1) and (4) are opposite to those of tracks (2) and (3). For a Type I vehicle, in Eq. (1), $l_{1 i}=l_{11}, \beta_{12}=\pi-\beta_{11}, \beta_{13}=$ $\pi+\beta_{11}, \beta_{12}=2 \pi-\beta_{11}, \theta_{11}=\theta_{13}=\pi, \theta_{12}=\theta_{14}=0, \alpha_{11}=$ $\alpha_{14}=\lambda_{1}$, and $\alpha_{12}=\alpha_{13}=-\lambda_{1}$, where $\lambda_{p} \in\left(0, \frac{\pi}{2}\right)$. Setting $B_{y \mathrm{pi}}=\left|l_{\mathrm{pi}} \sin \beta_{\mathrm{pi}}\right|$ and $B_{x \mathrm{pi}}=\left|l_{\mathrm{pi}} \cos \beta_{\mathrm{pi}}\right|, B_{y 1 i}=B_{y 11}$, $B_{x 1 i}=B_{x 11}$.

A Type II vehicle consists of four tracks, with two tracks arranged on each side of the vehicle body, and the roller offset angles of the same side track are opposite. For a Type II vehicle, in Eq. (1), $l_{21}=l_{23}, l_{22}=l_{24}, \beta_{22}=\beta_{21}=\pi$, $\beta_{23}=\beta_{24}=0, \theta_{21}=\theta_{22}=\pi, \theta_{23}=\theta_{24}=0, \alpha_{21}=\alpha_{23}=$ $\lambda_{2}, \alpha_{22}=\alpha_{23}=-\lambda_{2}, B_{y 2 i}=0, B_{x 21}=B_{x 23}$, and $B_{x 22}=$ $B_{x 24}$.

A Type III vehicle consists of several tracks that are evenly distributed around the geometric center of the vehicle body, and the roller offset angles of each track are equal. For a Type III vehicle, in Eq. (1), $l_{3 i}=l_{31}, \beta_{3 i}=\theta_{3 i}=(i-1) \frac{2 \pi}{n}$, and $\alpha_{3 i}=\lambda_{3}$.

In summary, the ideal center-point steering model (kinematic equations) of the three types of vehicles can be obtained from Eq. (1) as shown in Eq. (2):

$$
\omega r=\left\{\begin{array}{cc}
\left(B_{x}+B_{y} \cot |\alpha|\right) \omega_{z} & \text { Type I } \\
B_{x} \omega_{z} & \text { Type II, Type III }
\end{array} .\right.
$$

\section{Center-point steering analysis based on skid conditions}

To establish a motion correction model based on skid conditions (hereinafter referred to as the "correction model") for the smooth steering of a tracked omni-vehicle on a horizontal road surface, the following assumptions are made (Cheng et al., 2006, 2007).

1. The vehicle is symmetrical about its longitudinal and transverse planes, and the center of mass and the geometric center of the vehicle coincide.

2. The ground pressure of the track (roller) is uniformly distributed, regardless of the pressure changes of the roller when it approaches the ground and when it leaves the ground.

3. The vehicle has low-speed uniform steering, ignoring the influences of the centrifugal force and angular velocity changes.

4. The track is a uniform and flexible belt, regardless of the width and misalignment of the track.

5. The driving resistance coefficient of the vehicle does not change due to steering.

The following is an analysis of the center-point steering motion for Type I, II, and III vehicles.

\subsection{Analysis of center-point steering motion of Type I vehicle}

Taking track (1) of the Type I vehicle as an example, the point on the longitudinal symmetry axis of the track grounding section is selected and the center-point steering motion in four parts, as shown in Fig. 2a-d, is analyzed. The vehicle has a clockwise center-point steering motion, with clockwise as the positive direction. In Fig. $2 a-d$, the blue arrow represents the velocity vector, and the red arrow represents the force vector. The parameters, such as the amplitude and angle of each vector in Fig. 2, are slightly exaggerated for the convenience of presentation and do not represent the actual values. Taking the horizontal ground as the Earth absolute coordinate system $X O Y$, a follow-up coordinate system $x o y$ is established on the vehicle body, where point $o$ is the plane projection of the geometric center of the vehicle. $l_{z}$ is the straight line where the longitudinal symmetry axis of the track grounding section lies. $P_{p k}$ is the type $k$ grounding point between the rollers on track (1) of the $p$-type vehicle and the ground, and $k=0,1,2,3 . P_{p 0}$ is the grounding point in the ideal center-point steering motion. $P_{p 1}, P_{p 2}$, and $P_{p 3}$ are the grounding points in the center-point steering motion considering the slip. $P_{p 1}$ is the grounding point at which the degree of slippage is zero, $P_{p 2}$ the grounding point with an ordinate greater than $P_{p 1}$, and $P_{p 3}$ the grounding point with an ordinate smaller than $P_{p 1}$.

\subsubsection{Analysis of ideal center-point steering motion}

First, the ideal center-point steering motion, as shown in Fig. 2a, is analyzed. Point $P_{10}\left(-B_{x 11} B_{y 11}\right)$ is the geometric center of the ground plane of track (1). $l_{\mathrm{g}}$ is the straight line in the free rotation direction of the grounded roller, and it is the vertical line of the roller axis. $A_{13}$ is the intersection of $l_{z}$ and the $o x$ axis. The extension line of the roller axis direction passing point $P_{10}$ and the straight line where the $o x$ axis is located intersect at point $A_{12} . V_{x}$ is the relative velocity vector of the track, and the direction is along the negative direction of the $y$ axis. $\boldsymbol{V}_{q k}$ is the following velocity vector, which is perpendicular to the $\boldsymbol{o} \boldsymbol{P}_{k}$ vector. $\boldsymbol{V}_{j k}$ is the absolute velocity vector, which is perpendicular to the $\boldsymbol{A}_{11} \boldsymbol{P}_{1 k}$ vector. Due to the interaction between the ground and the grounded roller, a reverse force $\boldsymbol{F}_{1 k}$ vector is generated in the opposite direction of its absolute speed. Letting $\left|\boldsymbol{F}_{1 k}\right|=F_{\mu 1}\left(m_{1}, L_{11}\right)=\frac{\mu m_{1} g}{4 L_{11}} \mathrm{~d} y$, where $\mu$ is the steering resistance coefficient (Song et al., 2008, 2009), $m_{p}$ the mass of the $p$-type velocity, and $g$ the acceleration of gravity, it is noted that $M_{p k}$ and $T_{p k}$ are the steering resistance torque and the driving torque, respectively, of the $k$-type grounding point of track (1) of the $p$-type vehicle.

According to the derivation process (Zhang and Huang, 2015) of Eq. (1), it can be seen that Eq. (1) is only applicable to the geometric center point of the plane of the track grounding section, as shown in point $P_{10}$ in Fig. 2a. At this time, 


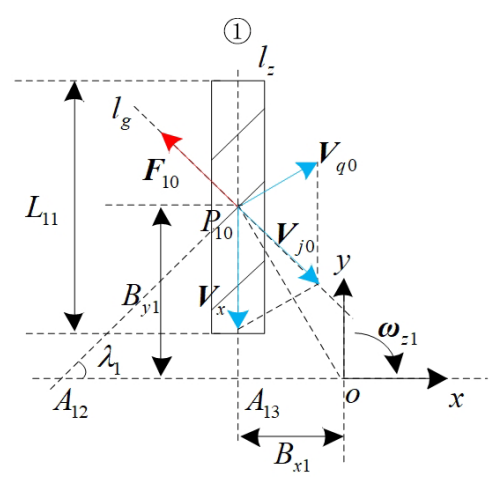

(a) Analysis of point $P_{10}$

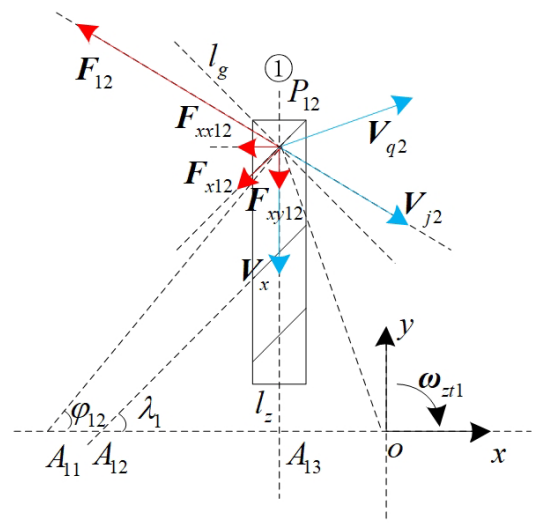

(c) Analysis of point $P_{12}$

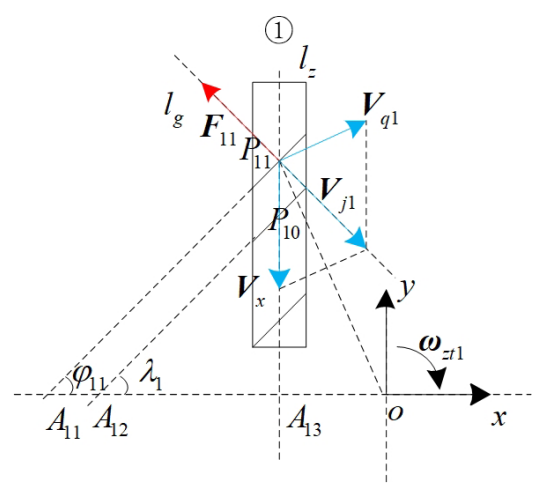

(b) Analysis of point $P_{11}$

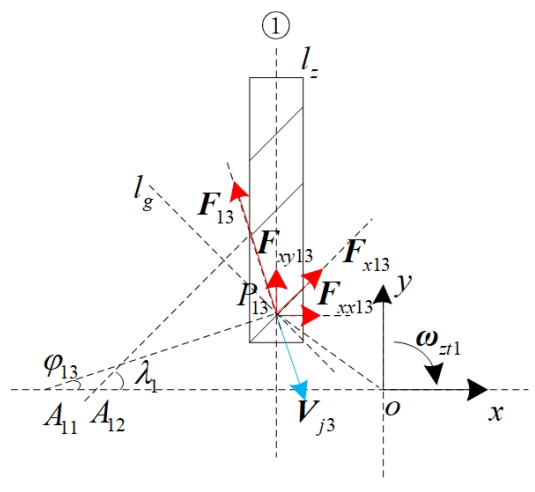

(d) Analysis of point $P_{13}$

Figure 2. Analysis of center-point steering motion of the Type I vehicle.

$\boldsymbol{V}_{j 0}$ and $l_{\mathrm{g}}$ are collinear, while $\boldsymbol{F}_{10}$ and $\boldsymbol{V}_{j 0}$ are collinear and opposite in direction. Because $\boldsymbol{V}_{j 0}$ is in the free-rotation direction of the roller, no slippage occurs. At this time, the effect of $\boldsymbol{F}_{10}$ on the roller is only the free rotation of the roller. Therefore, $A_{12}$ is the instantaneous turning center of the grounding section of track (1) without considering the slip.

In the ideal center-point steering motion, the instantaneous turning center of the left-hand track grounding section of a traditional dual-track longitudinally symmetrical tracked vehicle represented by a tank (hereinafter referred to as the "traditional tracked vehicle") is point $A_{13}$. The comparison shows that the steering pole of the Type I vehicle has an original lateral offset from $A_{13}$ to $A_{12}$ under ideal circumstances.

\subsubsection{Analysis of point at which slip is zero}

The movement of point $P_{11}\left(-B_{x 11} y_{0}\right)$ shown in Fig. $2 \mathrm{~b}$ is now analyzed. Because the track is slipping at this time, $\omega_{z 1}<\left|\omega_{z t 1}\right|$, where $\omega_{z t 1}$ represents the theoretical angular velocity vector of the center-point steering motion considering the slip. To cause $V_{j 1}$ to be in the free-rotation direction of the roller, $P_{11}$ must be above $P_{10}$. The extension line of the roller axis direction passing through point $P_{11}$ and the straight line of the $o x$ axis intersect at point $A_{11}\left(-A_{11}, 0\right)$. $A_{11}$ is the instantaneous turning center (turning pole) of the grounding section of track (1) considering slippage. $A_{\mathrm{pi}}$ is the absolute lateral offset distance of the steering pole. According to the symmetry, $A_{1 i}=A_{11} . l_{\mathrm{g}}$ is perpendicular to the line at which $A_{11} P_{11}$ is located. $\varphi_{1 k}$ is the acute angle between the line at which the $o x$ axis is located and that at which the $A_{11} P_{11}$ is located, and $\varphi_{1 k}>0$. At this time, $\varphi_{11}=\lambda_{1}$. Similar to the analysis in Fig. 2a, at this time, $\boldsymbol{V}_{j 1}$ and $l_{\mathrm{g}}$ are collinear, $\boldsymbol{F}_{11}$ and $\boldsymbol{V}_{j 1}$ are collinear and in opposite directions, and no slipping occurs. In addition, at this time, $M_{11}=T_{11}=0$.

\subsubsection{Analysis of upper point}

The movement of point $P_{12}$ shown in Fig. 2c is now analyzed. $\boldsymbol{V}_{j 2}$ is perpendicular to the $\boldsymbol{A}_{11} \boldsymbol{P}_{12}$ vector, and it lies on the right-hand half of $l_{\mathrm{g}} ; \varphi_{12}>\lambda_{1}$. The vector decomposition of $\boldsymbol{F}_{12}$ is conducted along the direction perpendicular to $l_{\mathrm{g}}$, and the decomposed vector is denoted as $\boldsymbol{F}_{12 x}$. 
$\boldsymbol{F}_{12 x}$ is decomposed along the $o x$ axis and $o y$ axis directions, and the decomposed vectors are denoted as $\boldsymbol{F}_{12 x x}$ and $\boldsymbol{F}_{12 x y}$, respectively. $\left|\boldsymbol{F}_{12 x}\right|=\left|\boldsymbol{F}_{12}\right| \sin \left(\varphi_{12}-\lambda_{1}\right),\left|\boldsymbol{F}_{12 x x}\right|=$ $\left|\boldsymbol{F}_{12 x}\right| \cos \lambda_{1}$, and $\left|\boldsymbol{F}_{12 x y}\right|=\left|\boldsymbol{F}_{12 x}\right| \sin \lambda_{1}$. In summary, $M_{12}=-\int_{y_{0}}^{y_{\max }}\left|\boldsymbol{F}_{12 x x}\right| y$ and $T_{12}=-\int_{y_{0}}^{y_{\max }}\left|\boldsymbol{F}_{12 x y}\right| B_{x 11}$ can be obtained, where $y_{0}=\left(A_{11}-B_{x 11}\right) \tan \lambda_{1}$ and $y_{\max }=$ $B_{y 11}+\frac{L_{11}}{2}$.

\subsubsection{Analysis of lower point}

The movement of point $P_{13}$ shown in Fig. 2d is now analyzed. $\boldsymbol{V}_{j 3}$ is perpendicular to the $\boldsymbol{A}_{11} \boldsymbol{P}_{13}$ vector, and it lies on the left-hand half of $l_{\mathrm{g}} ; \varphi_{13}<\lambda_{1}$. The vector decomposition of $\boldsymbol{F}_{13}$ is carried out along the direction of $l_{\mathrm{g}}$, and the decomposed vector is denoted as $\boldsymbol{F}_{13 x} . \boldsymbol{F}_{13 x}$ is decomposed along the $o x$ axis and $o y$ axis directions, and the decomposed vectors denote $\boldsymbol{F}_{13 x x}$ and $\boldsymbol{F}_{13 x y}$, respectively. $\left|\boldsymbol{F}_{13 x}\right|=\left|\boldsymbol{F}_{13}\right| \sin \left(-\varphi_{13}+\lambda_{1}\right),\left|\boldsymbol{F}_{13 x x}\right|=\left|\boldsymbol{F}_{13 x}\right| \cos \lambda_{1}$, and $\left|\boldsymbol{F}_{13 x y}\right|=\left|\boldsymbol{F}_{13 x}\right| \sin \lambda_{1}$. In summary, $M_{13}=\int_{y_{\min }}^{y_{0}}\left|\boldsymbol{F}_{13 x x}\right| y$ and $T_{13}=\int_{y_{\min }}^{y_{0}}\left|\boldsymbol{F}_{13 x y}\right| B_{x 11}$ can be obtained, where $y_{\min }=$ $B_{y 11}-\frac{L_{11}}{2}$.

\subsubsection{Synthesis}

Synthesizing the analysis of the above four parts and setting $\sin \varphi_{\mathrm{pi}}=\frac{y}{\sqrt{y^{2}+\left(A_{\mathrm{pi}}-B_{x p i}\right)^{2}}} \quad$ and $\quad \cos \varphi_{\mathrm{pi}}=\frac{A_{\mathrm{pi}}-B_{x p i}}{\sqrt{y^{2}+\left(A_{\mathrm{pi}}-B_{x p i}\right)^{2}}}$, the steering resistance torque is $M_{1}=M_{12}+M_{13}=$ $\int_{y_{\min }}^{y_{\max }} y \cos \lambda_{1} \sin \left(\lambda_{1}-\varphi_{1}\right) F_{\mu 1}$ and the driving torque is $T_{1}=T_{12}+T_{13}=\int_{y_{\min }}^{y_{\max }} B_{x 11} \sin \lambda_{1} \sin \left(\lambda_{1}-\varphi_{1}\right) F_{\mu 1}$. Letting $a_{\mathrm{pi}}$ be the relative lateral offset distance of the steering pole, $a_{\mathrm{pi}}=A_{\mathrm{pi}}-B_{x \mathrm{pi}}$. For a Type I vehicle, $a_{1 i}=a_{11}$. Letting $f_{T \text { pi }}$ be the total torque of the grounding section of track $i$ of a $p$-type vehicle, and given the initial conditions $a_{\mathrm{pi}}>0$ and $y_{\min }>0$, then

$$
\begin{aligned}
f_{T 11} & =\frac{\mu m_{1} g}{16 L_{11}}\left\{\operatorname { c o s } ^ { 2 } \lambda _ { 1 } \left[2 B_{y 11}\left(c_{1}-c_{2}\right)\right.\right. \\
& \left.-B_{y 11}\left(c_{1}+c_{2}\right)-2 a_{11}^{2} \ln \frac{c_{1}+y_{\min }}{c_{2}+y_{\max }}\right] \\
& -4 B_{x 11} a_{11} \ln \frac{c_{1}+y_{\min }}{c_{2}+y_{\max }} \sin ^{2} \lambda_{1} \\
& \left.-2\left(c_{1}-c_{2}\right)\left(a_{11}-B_{x 11}\right) \sin 2 \lambda_{1}\right\},
\end{aligned}
$$

where $c_{1}=\sqrt{a_{11}^{2}+y_{\min }^{2}}$ and $c_{2}=\sqrt{a_{11}^{2}+y_{\max }^{2}}$.

In the Type I vehicle, the analysis of the steering resistance and driving torque of the grounding section of tracks (2)-(4) is similar to that of track (1), and the expressions have the same form. The derivation process is omitted here. Letting $f_{z p}$ be the total center-point steering torque of $p$-type vehicle, then

$f_{z 1} \equiv \sum_{i=1}^{4} f_{T 1 i}=4 f_{T 11}$.

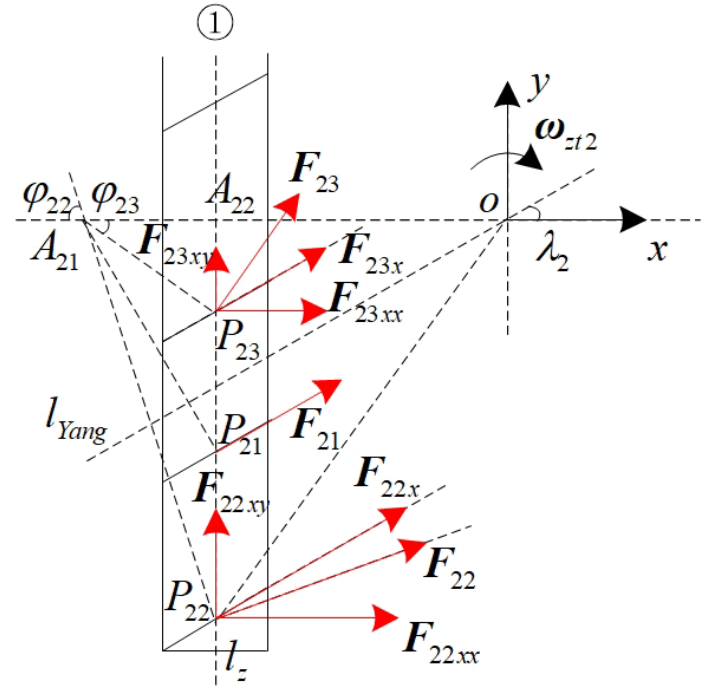

Figure 3. Analysis of center-point steering motion of Type II vehicle.

\subsection{Analysis of center-point steering motions of Type II and III vehicles}

First, the Type II vehicle is analyzed. The analysis of the center-point steering motion of the Type II vehicle based on skid conditions is similar to that of the Type I vehicle. Here, track (1) is again taken as an example for analysis, as shown in Fig. 3. For brevity, the expression and the analysis of the velocity vector are omitted here, and only the force vector is expressed and analyzed. In Fig. 3, only the lower half of the grounding section of track (1) is analyzed, because the analysis of the upper half is the same as that of the grounding section of track (1) of the Type I vehicle. The establishment of the coordinate system is the same as that in Sect. 3.1. Point $A_{22}$ is the intersection of $l_{z}$ and the $o x$ axis. Point $A_{21}\left(-A_{21}, 0\right)$ is the instantaneous steering center of the center-point steering motion based on skid conditions. $l_{\text {Yang }}$ is the design criterion line of the Type II vehicle track proposed by Yang et al. (2019b). The acute angle between $l_{\text {Yang }}$ and the $o x$ axis is equal to the roller offset angle. Yang et al. (2019b) pointed out that a design should follow the rule that the end point of the track (roller) grounding sec-

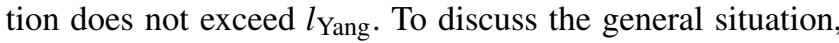
this rule is ignored in the following discussion. At this time, $\left|\boldsymbol{F}_{2 k}\right|=F_{\mu 2}=F_{\mu 1}\left(m_{2}, 2 H\right)=\frac{\mu m_{2} g}{8 H} d y$, where $H$ is half of the length of the track grounding section, $H=\frac{1}{2} L_{21}$.

$A_{22}\left(-B_{x 21}, 0\right)$ in Fig. 3 is the instantaneous steering center of track (1) of the grounding section of the ideal centerpoint steering motion. Letting $\varphi_{2 k}$ be the acute angle between the $o x$ axis and the line where $A_{21} P_{2 \mathrm{k}}$ is, $\varphi_{2 k}>0$. Then, $\varphi_{21}=\frac{\pi}{2}-\lambda_{2}$ (for brevity, this is not marked in Fig. 3), $\varphi_{22}>\frac{\pi}{2}-\lambda_{2}$, and $\varphi_{23}<\frac{\pi}{2}-\lambda_{2}$. The vector decomposition of $\boldsymbol{F}_{2 k}$ is conducted along the direction of the roller axis, and the decomposed vector denotes $\boldsymbol{F}_{2 k x} . \boldsymbol{F}_{2 k x}$ is decom- 
posed along the $o x$ axis and $o y$ axis directions, and the decomposed vectors denote $\boldsymbol{F}_{2 k x x}$ and $\boldsymbol{F}_{2 k x y}$. Additionally, $\left|\boldsymbol{F}_{2 k x}\right|=\left|\boldsymbol{F}_{2 k}\right| \cos \left(\frac{\pi}{2}-\lambda_{2}-\varphi_{2 k}\right), \quad\left|\boldsymbol{F}_{2 k x x}\right|=\left|\boldsymbol{F}_{2 k x}\right| \cos \lambda_{2}$, and $\left|\boldsymbol{F}_{2 k x y}\right|=\left|\boldsymbol{F}_{2 k x}\right| \sin \lambda_{2}$.

Letting $M_{2 k}$ be the steering resistance torque and letting $T_{2 k}$ be the driving torque, then $\sum_{k=1}^{3} M_{2 k}=$ $-\int_{-H}^{0}-y \cos \lambda_{2} \sin \left(\lambda_{2}+\varphi_{2}\right) F_{\mu 2} \quad$ and $\quad \sum_{k=1}^{3} T_{2 k}=$ $\int_{-H}^{0} B_{x 21} \sin \lambda_{2} \sin \left(\lambda_{2}+\varphi_{2}\right) F_{\mu 2}$. Analyzing the entire track grounding section (1) of the Type II vehicle, the total steering resistance torque is $M_{2}=-\int_{0}^{H} 2 y \cos ^{2} \lambda_{2} \sin \varphi_{2} F_{\mu 2}$ and the total driving torque is $T_{2}=\int_{0}^{H} B_{x 21} \sin ^{2} \lambda_{2} \cos \varphi_{2} F_{\mu 2}$. Given the initial conditions $H>0$, the expression of the total torque of the grounding section of track (1) of the Type II vehicle is

$$
\begin{aligned}
f_{T 21}= & -\frac{\mu m_{2} g}{8 H}\left[\operatorname { c o s } ^ { 2 } \lambda _ { 2 } \left(H \sqrt{a_{21}^{2}+H^{2}}\right.\right. \\
& \left.+a_{21}^{2} \ln \frac{a_{21}}{H+\sqrt{a_{21}^{2}+H^{2}}}\right) \\
& \left.+2 B_{x 21} a_{21} \sin ^{2} \lambda_{2} \ln \frac{a_{21}}{H+\sqrt{a_{21}^{2}+H^{2}}}\right] .
\end{aligned}
$$

The analysis of the steering resistance torque and the driving torque of the grounding section of tracks (2)-(4) is similar to that of track (1), and here,

$$
\begin{aligned}
f_{T 22}= & -\frac{\mu m_{2} g}{8 H}\left[\operatorname { c o s } ^ { 2 } \lambda _ { 2 } \left(H \sqrt{a_{22}^{2}+H^{2}}\right.\right. \\
& \left.+a_{22}^{2} \ln \frac{a_{22}}{H+\sqrt{a_{22}^{2}+H^{2}}}\right) \\
& \left.+2 B_{x 22} a_{22} \sin ^{2} \lambda_{2} \ln \frac{a_{22}}{H+\sqrt{a_{22}^{2}+H^{2}}}\right] .
\end{aligned}
$$

For the Type II vehicle, $A_{21}=A_{23}, A_{22}=A_{24}, a_{21}=a_{23}$, $a_{22}=a_{24}, f_{T 21}=f_{T 23}$, and $f_{T 22}=f_{T 24}$.

It can be seen from the above analysis that the two example layouts of Type II shown in Table 1 are equivalent in the center-point steering analysis. The overall center-point steering torque of the Type II vehicle can be expressed as

$f_{z 2} \equiv \sum_{i=1}^{4} f_{T 2 i}=2 f_{T 21}+2 f_{T 22}$.

The analysis of the center-point steering based on the skid conditions of the Type III vehicle is the same as that of the Type II vehicle. The first step of the analysis is to analyze a certain track and then extend the analysis results to other tracks. According to the central symmetry of the Type III layout, the expression of the total torque of the center-point steering of the Type III vehicle is

$f_{z 3} \equiv \sum_{i=1}^{n} f_{T 3 i}=n f_{T 31}$.
Supposing that the driving torque of the center-point steering motion of the traditional tracked vehicle is $T_{c}$, and the steering resistance torque is $M_{c}$, and comparing the expressions in Cheng et al. (2006), with the premise that the direction of the reference frame and the parameters are consistent,

$$
f_{z 2}=M_{c} \cos ^{2} \lambda_{2}+T_{c} \sin ^{2} \lambda_{2} \text {. }
$$

Equation (9) is consistent with the derivation process of the center-point steering motion formula of the Type II vehicle. It can be seen that due to the decomposition of the rollers on the force the driving torque and the steering resistance torque of the Type II vehicle are both the secondary components of the stress moment of the traditional tracked vehicle.

\section{Models and angular velocity correction}

Considering the driving resistance, a model of the centerpoint steering motion based on the skid conditions of the $p$-type vehicle is established. Substituting the specific structural parameters of the $p$-type vehicle and using the numerical solution to solve the equation to obtain $A_{\mathrm{pi}}, A_{\mathrm{pi}}$ is used to modify the ideal center-point steering motion equation of the $p$-type vehicle and to calculate the center-point steering angular velocity reduction coefficient of the $p$-type vehicle.

\subsection{Models of center-point steering motion based on skid conditions}

The three types of vehicles have a low-speed uniform centerpoint steering motion on level ground. According to the plane motion equation of a rigid body,

$f_{z p}+M_{r p}=0$,

where $M_{r p}$ is the driving resistance torque. $M_{r p}=$ $\frac{1}{N} m_{p} g \gamma \sum_{i=1}^{N} B_{x \mathrm{pi}}$, where $N$ is the number of tracks of the $p$-type vehicle and $\gamma$ the road resistance coefficient. To express Eq. (10) in the form of a function,

$f\left(\mu, B_{y \mathrm{pi}}, L_{p 1}, \lambda_{p}, A_{\mathrm{pi}}, B_{x \mathrm{pi}}, \gamma\right)=0$.

When the parameters $\mu, B_{y \mathrm{pi}}, L_{p 1}, \lambda_{p}, B_{x \mathrm{pi}}$, and $\gamma$ in Eq. (11) are known, the equation can be solved and $A_{\text {pi }}$ can be obtained. Equation (11) is a transcendental equation and an analytical solution cannot be obtained. A numerical iterative solution method is used to find $A_{\mathrm{pi}}$.

The roller material of the tracked omni-vehicle is generally hard rubber or polyurethane. The working environment of the tracked omni-vehicle is generally a factory workshop or an outdoor flat road, and a cement road is the common working road. In summary, the parameter values of the ground are set to $\mu=0.7$ and $\gamma=0.04$. An example containing specific parameters for each type of the tracked omni-vehicle is chosen, and the absolute lateral offset distance is calculated. Taking vehicles (1), (3), and (7) in Table 2 as examples, $A$ is calculated and a comparative analysis is performed. Table 2 shows 
the basic parameters of virtual prototypes (1)-(8), where $\Lambda$ is the length-to-width ratio of the track grounding section.

The relationship between the two sets of parameters $\{L, A\}$ and $\{\alpha, A\}$ of the virtual prototypes (1), (2), and (7) is calculated as shown in Fig. $4 a$ and $b$, where the blue curve is the average value of $A$ for the two tracks on the same side of virtual prototype (3). In engineering design, the design experience of $\alpha \in\left[\frac{\pi}{6}, \frac{\pi}{3}\right]$ is generally followed because a roller offset angle that is too small or too large will complicate the design and processing of track shoes, and it will affect the anisotropic distribution of the translational velocity and the acceleration of the omni-vehicle (Zhang and Huang, 2015; Yang et al., 2019b; Zhang et al., 2017). Therefore, $\alpha \in\left[\frac{\pi}{12}, \frac{5 \pi}{12}\right]$ is taken in Fig. $4 \mathrm{~b}$.

It can be seen from Fig. 4 that $A$ increases as $L$ increases and $A$ decreases as $\alpha$ increases. The $A$ value of the Type II and III vehicles is more affected by the change of $\alpha$ and that of the Type I vehicle is less affected. Using a similar drawing method to analyze the relationship between $B_{y} A, B_{x} A$, and $B_{x} a$ of the three vehicles, it can be seen that $A$ increases with the increasing $B_{y}$ and $B_{x}$, and $a$ decreases with the increasing $B_{x}$.

\subsection{Angular velocity correction of center-point steering motion}

The theoretical center-point steering angular velocity $\omega_{z t}$ of the three vehicles based on skid conditions can be obtained according to the following formula:

$\omega r=A \omega_{z t}$.

Combining Eqs. (2) and (12), the ratio of $\omega_{z t}$ to $\omega_{z}$ can be obtained as shown in Eq. (13):

$\frac{\omega_{z t}}{\omega_{z}}=\left\{\begin{array}{cc}\frac{B_{x}+B_{y} \cot |\alpha|}{A} & \text { Type I } \\ \frac{B_{x}}{A} & \text { Type II, Type III }\end{array}\right.$.

The descent coefficient function is defined as $\psi\left(x_{1}, x_{2}\right)=\frac{x_{1}-x_{2}}{x_{2}}, \quad$ and $\quad \psi_{z t}=-\psi\left(\omega_{z t}, \omega_{z}\right), \quad \psi_{z s}=$ $\psi\left(\omega_{z}, \omega_{z s}\right), \quad \psi_{t s}=\psi\left(\omega_{z t}, \omega_{z s}\right), \quad \psi_{z r}=\psi\left(\omega_{z}, \omega_{z r}\right)$, and $\psi_{t r}=\psi\left(\omega_{z t}, \omega_{z r}\right)$, where $\omega_{z s}$ is the average value of the center-point steering angular velocity of the virtual prototype in the simulation and $\omega_{z r}$ is the average value of the center-point steering angular velocity of the physical prototype in the experiment. $\psi_{s}=\left|\psi_{z s}\right|-\left|\psi_{t s}\right|$ is the steering angular velocity reduction coefficient of the virtual prototype before and after correction, and $\psi_{r}=\left|\psi_{z r}\right|-\left|\psi_{t r}\right|$ is the steering angular velocity reduction coefficient of the physical prototype before and after correction.

Taking the virtual prototypes (1), (3), and (7) in Table 2 as examples, the relationship curve between $\psi_{z t}$ and $\alpha$ is drawn as shown in Fig. 5, from which it can be seen that $\psi_{z t}$ always decreases with increasing $\alpha$. It can be seen from Fig. 5 that in the Type II and III vehicles, $\psi_{z t}$ is more sensitive to changes in $\alpha$. When $\alpha \in\left[\frac{\pi}{6}, \frac{\pi}{3}\right]$, the $\psi_{z t}$ value of virtual prototype (1) is always smaller than that of virtual prototypes (3) and (7). When $\alpha \in\left[\frac{\pi}{12}, \frac{5 \pi}{12}\right]$, the $\psi_{z t}$ value of virtual prototype (7) is always smaller than that of virtual prototype (3). This is because the ratio $\frac{B_{y}}{B_{x}}$ of virtual prototype (7) is smaller than that of virtual prototype (3).

According to the analysis of the translational velocity and the acceleration anisotropy of the tracked omni-vehicle (Zhang and Huang, 2015; Fang et al., 2020; Yang et al., 2019b; Zhang et al., 2017), the optimal value of the roller offset angle in translational motion is $\frac{\pi}{4} \mathrm{rad}$. For the Type I vehicle, the roller offset angle is set to $\frac{\pi}{4} \mathrm{rad}$, and this can take into account the best translational motion and center steering motion performance. For the Type II and III vehicles, the offset angle of $\frac{\pi}{4}$ rad will cause serious slippage in the center-point steering. Therefore, the offset angle should be greater than $\frac{\pi}{4} \operatorname{rad}$. Additionally, the general value is $\frac{\pi}{3}$ $\mathrm{rad}$. At this time, the optimal translational performance cannot be considered.

\section{Center-point steering analysis based on skid conditions}

According to the design parameters in Table 2, virtual prototypes (1)-(8) are established in ADAMS, and the centerpoint steering motion simulation is performed. The correction model is applied to correct $\omega_{z} . \psi_{z s}, \psi_{t s}$ and $\psi_{s}$ are calculated, and the calculation results are compared and analyzed. Physical prototypes corresponding to the virtual prototypes (1), (2), and (4) are established, and center-point steering motion experiments are conducted. $\psi_{z r}, \psi_{t r}$, and $\psi_{r}$ are calculated, and the calculation results are compared and analyzed.

\subsection{Simulation and analysis}

Virtual prototypes established in ADAMS based on Table 2 are shown in Fig. 6.

In the simulation, each driving wheel of the same vehicle is driven by the same form of STEP function. The STEP function is applied to the revolute joint of the driving wheel. The syntax of the STEP function is " $\operatorname{STEP}(x 0, x 1, h 1, x 2, h 2)$ ", where $x 0$ represents the independent variable, $x l$ represents the initial value of the independent variable, $h l$ represents the initial value of the STEP function, $x 2$ represents the end value of the independent variable, and $h 2$ represents the end value of the STEP function. The STEP functions of the eight virtual prototypes in the simulation are shown in the Table 3.

The angular velocity curve of the center-point steering of virtual prototypes (1)-(8) is shown in Fig. 7. The solid line in the figure represents the simulated angular velocity value of the virtual prototype measured. The dotted line represents the calculated $\omega_{z}$ value. A positive angular velocity indicates that the virtual prototype turns counterclockwise, and a negative angular velocity indicates that the virtual prototype turns 
Table 2. Basic parameters of virtual prototypes (1)-(8).

\begin{tabular}{|c|c|c|c|c|c|c|c|c|}
\hline Type & No. & $m(\mathrm{~kg})$ & $B_{y}(\mathrm{~mm})$ & $B_{x}(\mathrm{~mm})$ & $\alpha(\mathrm{rad})$ & $L(\mathrm{~mm})$ & $\Lambda$ & $r(\mathrm{~mm})$ \\
\hline \multirow{2}{*}{ I } & (1) & 5070 & 694 & 579 & \multirow[t]{2}{*}{$\pi / 4$} & 505 & 3.1 & 147.5 \\
\hline & (2) & 340 & 560 & 558 & & 610 & 5.5 & 173 \\
\hline \multirow{3}{*}{ II } & (3) & 92 & \multirow{2}{*}{0} & \multirow{2}{*}{$295 \quad 23$} & $\pi / 3$ & \multirow{2}{*}{700} & 23.3 & \multirow{2}{*}{112} \\
\hline & (4) & 82 & & & $\pi / 4$ & & 16.7 & \\
\hline & (5) & 180 & & 550 & & 698 & 16.6 & \\
\hline \multirow[t]{3}{*}{ III } & (6) & 108 & \multirow{3}{*}{0} & \multirow{3}{*}{685} & \multirow{3}{*}{$\pi / 4$} & 317 & 7.5 & \multirow{3}{*}{112} \\
\hline & (7) & 111 & & & & 698 & 16.6 & \\
\hline & (8) & 212 & & & & 1080 & 25.7 & \\
\hline
\end{tabular}

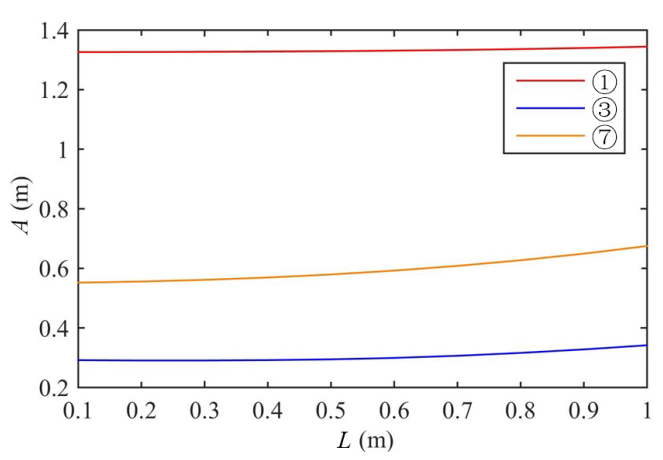

(a) Relationship lines of $\{L, A\}$

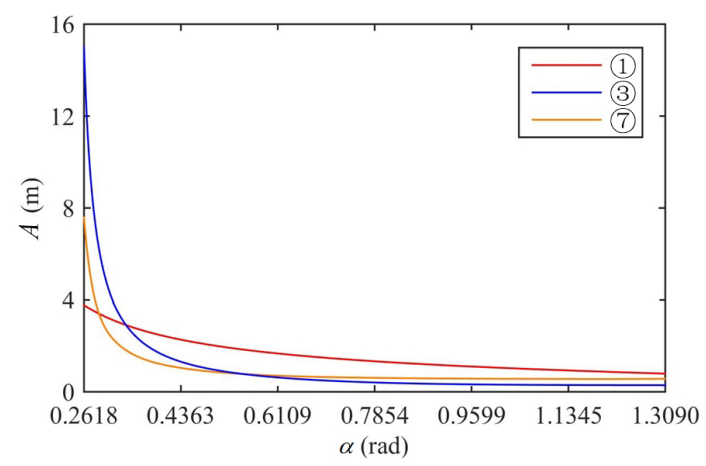

(b) Relationship lines of $\{\alpha, A\}$

Figure 4. Relationship lines.

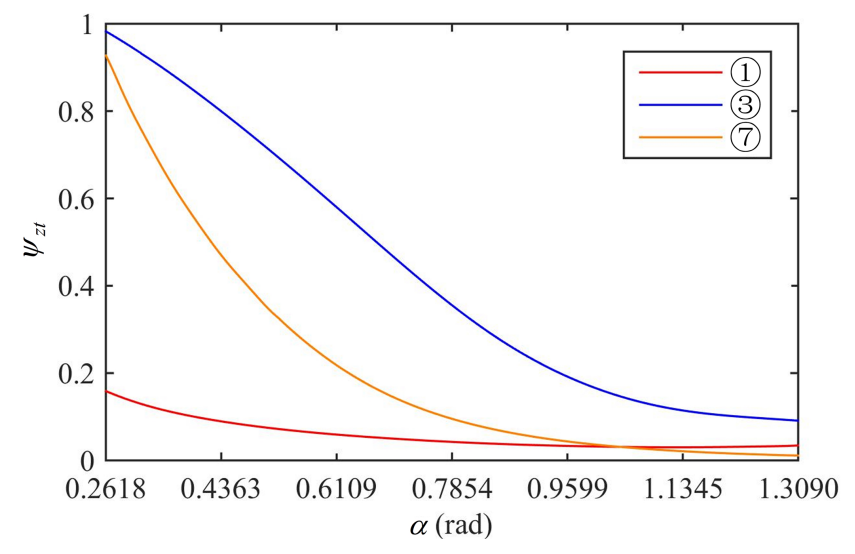

Figure 5. Relationship curves of $\psi_{z t}$ and $\alpha$.

clockwise. Comparing the solid and dashed lines in Fig. 7a$\mathrm{h}$, it can be seen that the simulated angular velocity values of virtual prototypes (3), (4), (5), and (8) are far from the ideal angular velocity values.

The parameters of the center-point steering of virtual prototypes (1)-(8) obtained by calculation are shown in Table 4 .
From the information about the virtual prototypes (1), (6), and (8) in Fig. 7 and Table 4, it can be seen that the difference in the speed of the driving wheels does not cause drastic changes in the values of parameters such as $\psi_{z s}$ and $\psi_{t s}$, which shows the correctness of the built simulation model and the modified model. It can be seen from Fig. 7 and Table 4 that the $\psi_{z s}$ values of virtual prototypes (1) and (2) are relatively small because of the low slippage characteristics of the Type I vehicle. The $\psi_{z s}$ value of virtual prototype (6) is small because the $L_{1}$ value of (6) (as shown in Table 2) is small.

Compared with $\psi_{s}$ in Table 4 , it can be seen that the correction effects of virtual prototypes (1), (2), and (6) are not good, but the corrections increase the absolute values of the errors. The correction effects of virtual prototypes (7) and (8) are not obvious, and the absolute values of the errors are slightly reduced after correction. The correction effects of virtual prototypes (3)-(5) are better, and the absolute value of the error is greatly reduced after correction. The reason for the above correction difference is that the $\Lambda$ values of virtual prototypes (1), (2), and (6) (as shown in Table 2) are small, which makes it impossible to ignore the influence of the track width on center-point steering, which contradicts the assumption (4) in Sect. 2. According to design experi- 


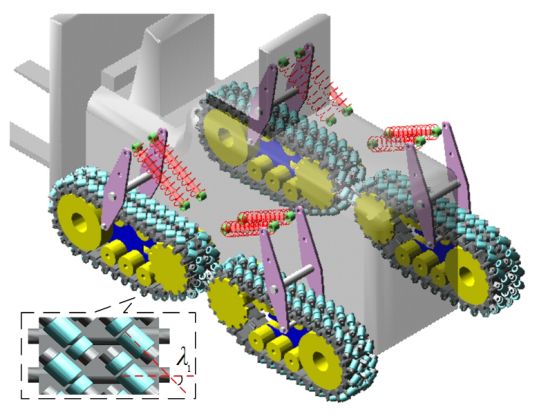

(a) Virtual prototype (1)

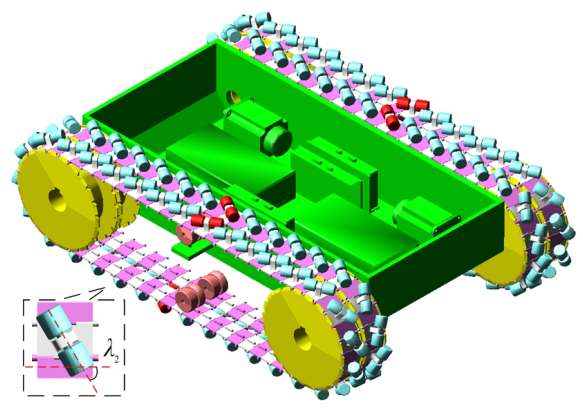

(c) Virtual prototype (3)

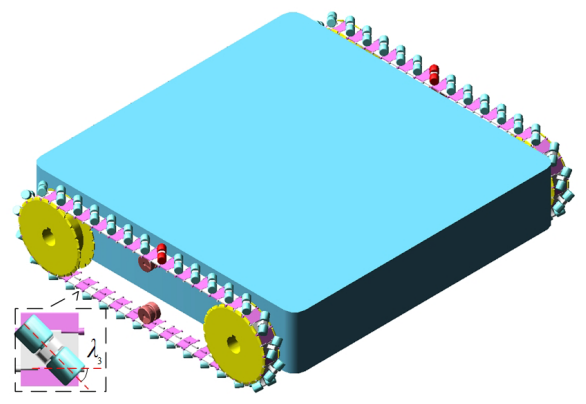

(e) Virtual prototype (5)

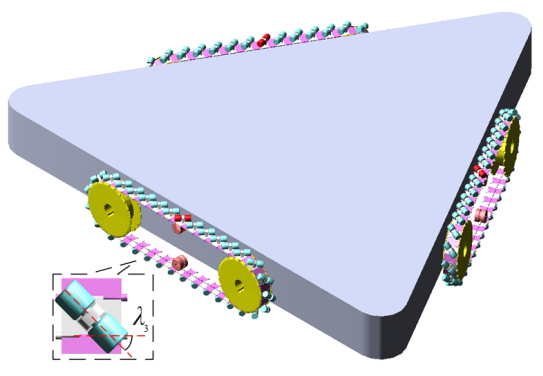

(g) Virtual prototype (7)

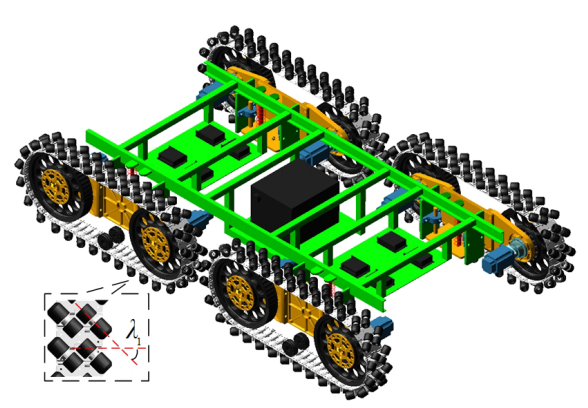

(b) Virtual prototype (2)

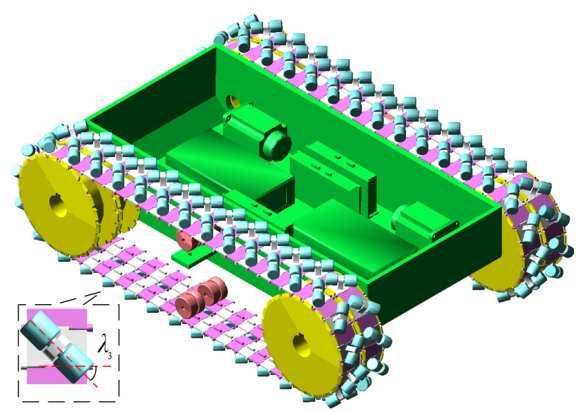

(d) Virtual prototype (4)

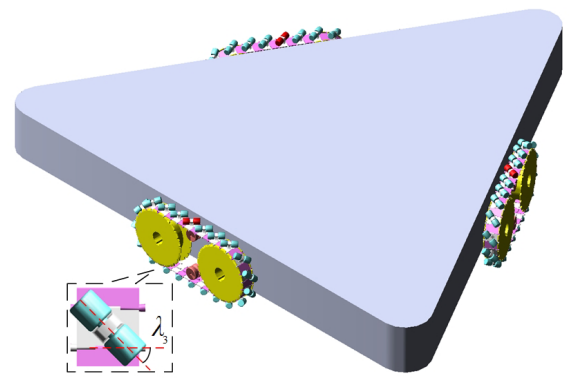

(f) Virtual prototype (6)

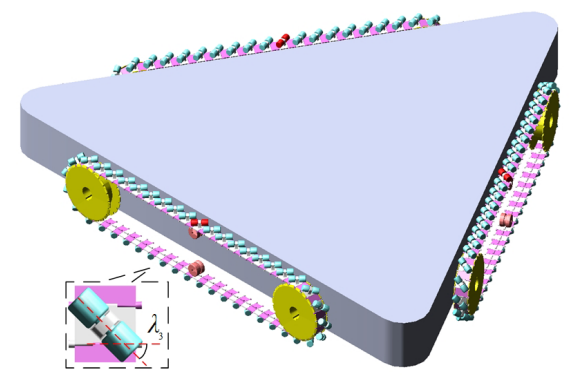

(h) Virtual prototype (8)

Figure 6. Virtual prototypes (1)-(8).

ence, the condition of the assumption (4) is $\Lambda>10$. From the perspective of the three types, it can be seen that the modified model is generally suitable for theoretical calculation and the correction of Type II and III vehicles, and it is generally not necessary to modify the Type I vehicle.

\subsection{Experiments and analysis}

Physical prototypes (1), (2), and (4) corresponding to the virtual prototypes (1), (2), and (4), respectively, in Table 2 and Fig. 6 are established and center-point steering motions are conducted, as shown in Fig. 8. The wheels of the physical 
Table 3. STEP functions of virtual prototypes (1)-(8).

\begin{tabular}{|c|c|c|c|}
\hline Type & Virtual prototype & $\omega_{z s}$ & STEP function \\
\hline \multirow{3}{*}{ I } & (1) & $\pi$ & STEP (time, 1,0,1.6,180d) \\
\hline & (1) & $2 \pi$ & STEP (time, $1,0,3,360 \mathrm{~d}$ ) \\
\hline & (2) & 6.37 & STEP (time, $0.5,0,2,6.37$ ) \\
\hline \multirow{2}{*}{ II } & (3) & \multirow{2}{*}{$\pi / 2$} & \multirow{2}{*}{ STEP (time, $0.5,0,1.5,90 \mathrm{~d}$ ) } \\
\hline & (4) & & \\
\hline \multirow{6}{*}{ III } & (5) & $2 \pi$ & STEP (time, $0.5,0,2,360 \mathrm{~d}$ ) \\
\hline & (6) & $\pi / 2$ & STEP (time, $0,0,0.5,90 \mathrm{~d}$ ) \\
\hline & (6) & \multirow{2}{*}{6.1161} & \multirow{2}{*}{ STEP (time, $0,0,0.5,6.116$ ) } \\
\hline & (7) & & \\
\hline & (8) & $\pi / 2$ & STEP (time, $0.5,0,1.5,90 \mathrm{~d}$ ) \\
\hline & (8) & $\pi$ & STEP (time, $0.5,0,1.5,180 \mathrm{~d}$ ) \\
\hline
\end{tabular}

Table 4. Calculation of center-point steering parameters of virtual prototypes (1)-(8).

\begin{tabular}{lllrrrrrr}
\hline Type & No. & $\omega\left(\mathrm{rad} \mathrm{s}^{-1}\right)$ & $\omega_{z}\left(\mathrm{rad} \mathrm{s}^{-1}\right)$ & $\omega_{z t}\left(\mathrm{rad} \mathrm{s}^{-1}\right)$ & $\omega_{z s}\left(\mathrm{rad} \mathrm{s}^{-1}\right)$ & $\psi_{z s}$ & $\psi_{t s}$ & $\psi_{s}$ \\
\hline \multirow{3}{*}{$\mathrm{I}$} & $(1)$ & $\pi$ & 0.3625 & 0.3471 & 0.359 & $0.97 \%$ & $-3.31 \%$ & $-2.34 \%$ \\
& $(1)$ & $2 \pi$ & 0.7305 & 0.6995 & 0.7225 & $1.11 \%$ & -3.18 & $-2.07 \%$ \\
& $(2)$ & 6.37 & 0.9857 & 0.9421 & 1.0227 & $-3.62 \%$ & $-7.88 \%$ & $-4.26 \%$ \\
\hline \multirow{2}{*}{ II } & $(3)$ & $\pi / 2$ & 0.6702 & 0.5947 & 0.5646 & $18.70 \%$ & $5.34 \%$ & $13.36 \%$ \\
& $(4)$ & & & 0.4393 & 0.4503 & $48.83 \%$ & $-2.44 \%$ & $46.39 \%$ \\
\hline & $(5)$ & $2 \pi$ & 1.2566 & 1.0889 & 1.0982 & $14.42 \%$ & $-0.85 \%$ & $13.57 \%$ \\
& $(6)$ & $\pi / 2$ & 0.2568 & 0.2519 & 0.2618 & $-1.91 \%$ & $-3.77 \%$ & $-1.86 \%$ \\
III & $(6)$ & 6.1161 & 1 & 0.9810 & 1.01 & $-0.99 \%$ & $-2.87 \%$ & $-1.88 \%$ \\
& $(7)$ & & 1 & 0.9272 & 0.96 & $4.17 \%$ & $-3.42 \%$ & $0.75 \%$ \\
& $(8)$ & $\pi / 2$ & 0.2568 & 0.2371 & 0.2331 & $10.17 \%$ & $1.70 \%$ & $8.47 \%$ \\
& $(8)$ & $\pi$ & 0.5137 & 0.4742 & 0.4617 & $11.26 \%$ & $2.71 \%$ & $8.55 \%$ \\
\hline
\end{tabular}

prototypes (1), (2), and (4) are all hard rubber, and the road conditions are all dry cement roads. The center-point steering process of physical prototypes (1) and (2) is smooth. In the process of center-point steering, the shift of the steering center of physical prototype (4) is more obvious, and the tracks near the vehicle body vibrated more than those far from the vehicle body do, and the phenomenon of blocking in motion manifests.

According to Eqs. (2), (12), and (13), $\omega_{z}, \omega_{z t}, \psi_{z r}, \psi_{t r}$, and $\psi_{r}$ are calculated as shown in Table 5. Compared with $\psi_{r}$, it can be seen that the correction model has a positive correction effect on physical prototypes (1) and (4), reducing the speed error, and it has a significant correction effect on physical prototype (4). The correction model has a negative correction effect on physical prototype (2), but it increases the speed error. The conclusion obtained with the analysis of $\psi_{r}$ is consistent with the conclusion obtained with the analysis of $\psi_{s}$. It can be seen that the correction model and the virtual prototypes are correct.

\section{Conclusions}

Taking three typical layouts as examples, in this study, the center-point steering motion of a tracked omni-vehicle based on skid conditions is analyzed, and the analysis results are compared and analyzed using the simulation and experimental data. The following conclusions are drawn.

1. Compared with traditional tracked vehicles, the rectangular layout of a tracked omni-vehicle has a horizontal original offset of the steering pole in the center-point steering motion. This original offset is related to the roller offset angle and the track grounding length of the vehicle. The parameters are directly related.

2. In the center-point steering motion, due to the force decomposition of the rollers, the driving torque and the steering resistance torque of the Type II and III vehicles are the secondary components of the corresponding torque of the traditional tracked vehicle.

3. For the case of the same roller offset angle, in centerpoint steering, the slips of the Type II and III vehicles 


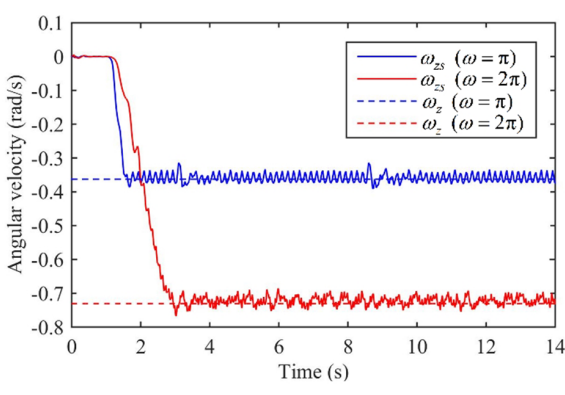

(a) Angular velocity curve of virtual prototype (1)

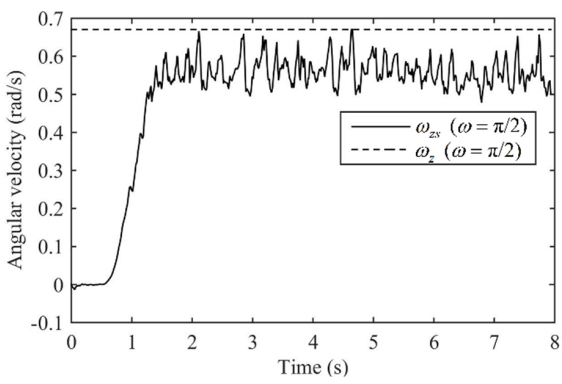

(c) Angular velocity curve of virtual prototype (3)

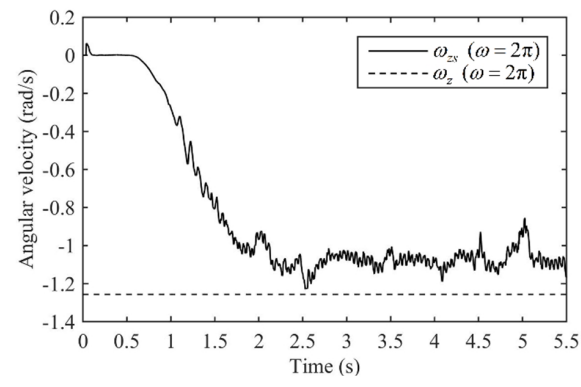

(e) Angular velocity curve of virtual prototype (5)

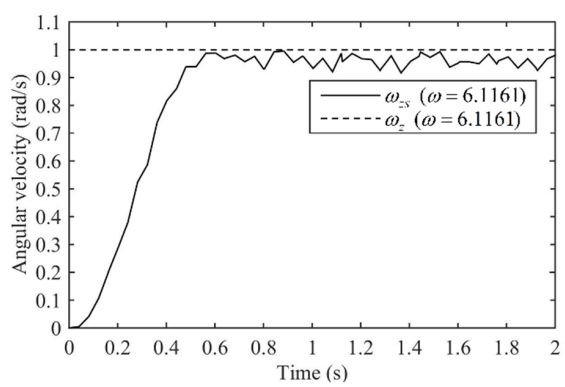

(g) Angular velocity curve of virtual prototype (7)

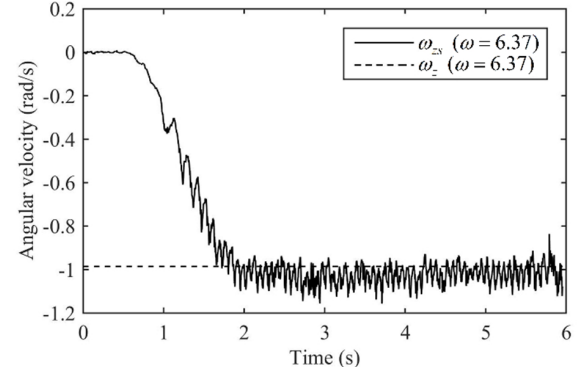

(b) Angular velocity curve of virtual prototype (2)

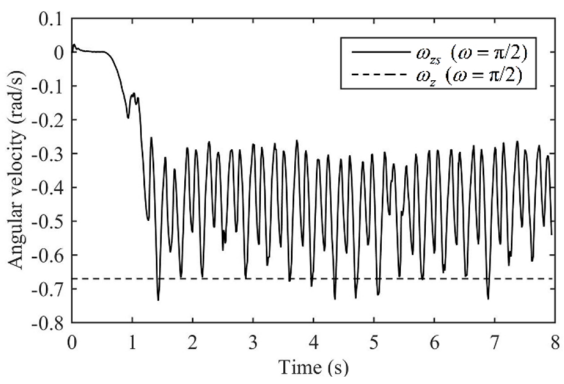

(d) Angular velocity curve of virtual prototype (4)

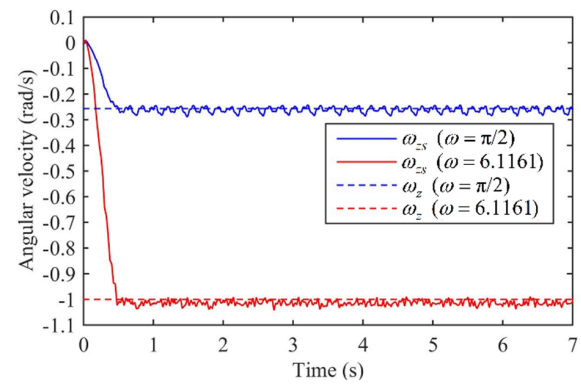

(f) Angular velocity curve of virtual prototype (6)

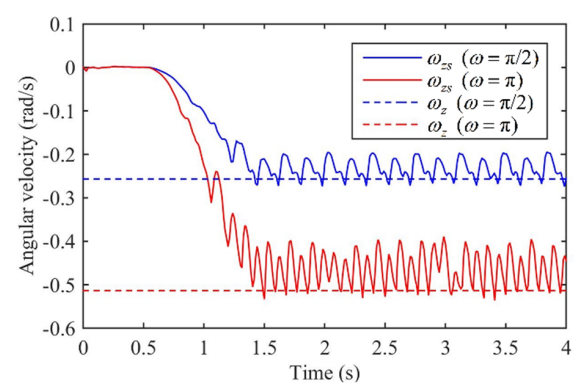

(h) Angular velocity curve of virtual prototype (8)

Figure 7. Center-point steering angular velocity curve of virtual prototypes (1)-(8).

are more serious than that of the Type I vehicle. When higher requirements for the center-point steering exist in the design stage, the Type I vehicle should be selected for structural design first. When comprehensively considering the translation and center-point steering motion performance, the optimal value of the roller offset angle of the Type I vehicle is generally $\frac{\pi}{4} \mathrm{rad}$. The optimal value of the roller offset angle of the hybrid and centripetal layout vehicles should generally be greater than $\frac{\pi}{4} \mathrm{rad}$, and $\frac{\pi}{3} \mathrm{rad}$ is usually chosen as a compromise. 


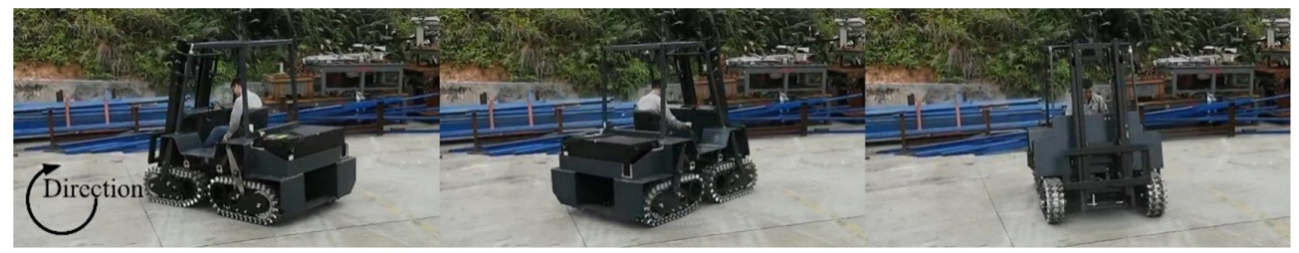

(a) Center-point steering motion of physical prototype (1)

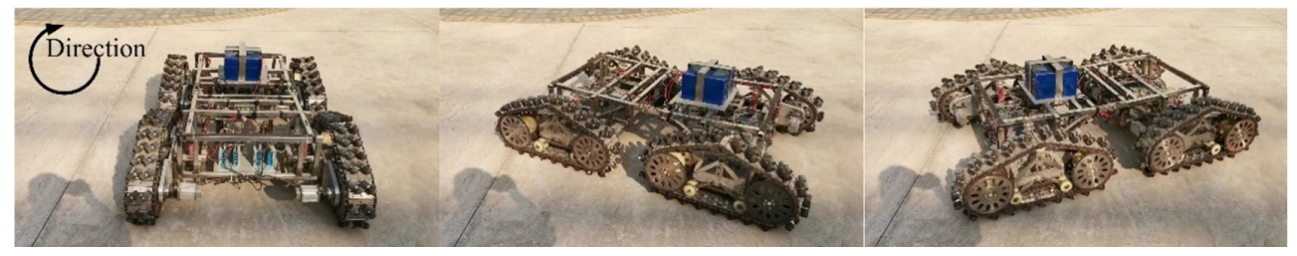

(b) Center-point steering motion of physical prototype (2)

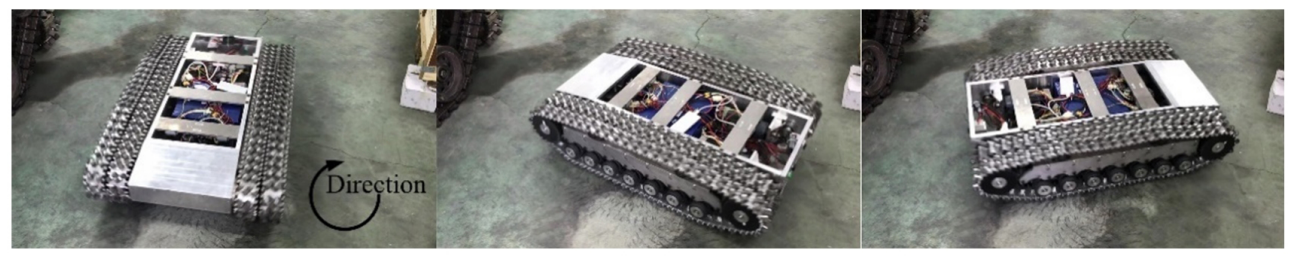

(c) Center-point steering motion of physical prototype (4)

Figure 8. Center-point steering motions of physical prototypes (1), (2), and (4).

Table 5. Data for center-point steering motion of physical prototypes.

\begin{tabular}{lrrrrrrr}
\hline Type & No. & $\omega_{z}\left(\mathrm{rad} \mathrm{s}^{-1}\right)$ & $\omega_{z t}\left(\mathrm{rad} \mathrm{s}^{-1}\right)$ & $\omega_{z r}\left(\mathrm{rad} \mathrm{s}^{-1}\right)$ & $\psi_{z r}$ & $\psi_{t r}$ & $\psi_{r}$ \\
\hline I & $(1)$ & 2.2260 & 2.1315 & 2.0944 & $6.28 \%$ & $1.77 \%$ & $4.51 \%$ \\
& $(2)$ & 1.1270 & 1.0772 & 1.1706 & $-3.72 \%$ & $-7.98 \%$ & $-4.26 \%$ \\
\hline II & $(4)$ & 1.5324 & 1.0045 & 0.9887 & $54.99 \%$ & $1.60 \%$ & $53.39 \%$ \\
\hline
\end{tabular}

4. The analysis of the simulation and experimental data shows that the reduction coefficient of the center-point steering angular velocity of the tracked omni-vehicle given in this article can play a corrective role. The correction effect on the Type II and III vehicles is more obvious than that of the Type I vehicle. The corrective effect is obvious when the length-to-width ratio of the track grounding is relatively large.

Based on the research in this article, the following four directions are worthy of further research in the future.

1. Researchers should consider studying equations of motion such as Eq. (7) from the perspective of force and power, e.g., by analyzing the relationship between the power of the vehicle's driving wheel and center-point steering radius, and obtaining the law of the vehicle's steering power.
2. Researchers should consider reducing the number of assumptions in Sect. 3 and conduct a more detailed study on the center-point steering motion of the tracked omni-vehicle. For example, researchers do not ignore the width of the track grounding section or study the center-point steering motion when the position of the center of gravity changes.

3. Researchers should consider the influence of the ground and soil on the center-point steering motion, explore the vehicle's moving capabilities on unstructured roads, and expand the application environment of the vehicle.

4. Different degrees of velocity and trajectory deviations will occur in the center-point steering motion. It is necessary to study the control methods to correct this deviation and find the control laws and methods to improve the steering performance. 


\section{Appendix A: List of symbols}

a relative offset distance of steering pole (m)

$A \quad$ absolute lateral offset distance of steering pole (m)

$B_{x}, B_{y} \quad$ length of projection of distance between center of track and center of vehicle in the $x$ axis and $y$ axis directions $(\mathrm{m})$

$f_{\mathrm{T}} \quad$ total torque of grounding section of track $(\mathrm{N} \mathrm{m})$

$f_{z} \quad$ total center-point steering torque $(\mathrm{N} \mathrm{m})$

$\boldsymbol{F} \quad$ reverse force vector

$\boldsymbol{F}_{x} \quad$ component force of $\boldsymbol{F}$ along axis of roller

$\boldsymbol{F}_{x x} \quad$ component force of $\boldsymbol{F}_{x}$ along $o x$ axis direction

$\boldsymbol{F}_{x y} \quad$ component force of $\boldsymbol{F}_{x}$ along oy axis direction

$g \quad$ acceleration due to gravity $\left(\mathrm{m} \mathrm{s}^{-2}\right)$

$H \quad$ half of length of track grounding section (m)

$\mathbf{J}_{\omega} \quad$ Jacobian matrix

$l \quad$ distance between center of track and center of vehicle $(\mathrm{m})$

$L \quad$ length of single track grounding section (m)

$m \quad$ vehicle mass $(\mathrm{kg})$

$M \quad$ steering resistance torque $(\mathrm{N} \mathrm{m})$

$M_{c} \quad$ steering resistance torque of traditional tracked vehicle $(\mathrm{N} \mathrm{m})$

$M_{\mathrm{r}} \quad$ resistance moment during driving $(\mathrm{N} \mathrm{m})$

$N \quad$ number of tracks of $p$-type vehicle

$r \quad$ radius of driving wheel $(\mathrm{m})$

$T \quad$ steering driving torque $(\mathrm{N} \mathrm{m})$

$T_{c} \quad$ steering driving torque of traditional tracked vehicle $(\mathrm{N} \mathrm{m})$

$v_{x} \quad$ translational velocity in $x$ axis direction $\left(\mathrm{m} \mathrm{s}^{-1}\right)$

$v_{y} \quad$ translational velocity in $y$ axis direction $\left(\mathrm{m} \mathrm{s}^{-1}\right)$

$\boldsymbol{V}_{j} \quad$ absolute velocity vector

$\boldsymbol{V}_{q} \quad$ following velocity vector

$\boldsymbol{V}_{x} \quad$ relative velocity vector

\section{Greek symbols}

$\alpha \quad$ roller offset angle (rad)

$\beta \quad$ angle between center of track and center of vehicle (rad)

$\gamma \quad$ road resistance coefficient

$\eta \quad$ angle between roller axis and vehicle coordinate system ( $\mathrm{rad})$

$\theta \quad$ angle between coordinate system of track and coordinate system of vehicle (rad)

$\lambda \quad$ absolute value of roller offset angle (rad)

$\Lambda \quad$ length-to-width ratio of track grounding section

$\mu \quad$ steering resistance coefficient

$\varphi \quad$ acute angle between $o x$ axis and straight line connecting point on ground roller and instantaneous steering center (rad)

$\psi \quad$ descent coefficient function

$\psi_{r} \quad$ steering angular velocity reduction coefficient of physical prototype before and after correction

$\psi_{s} \quad$ steering angular velocity reduction coefficient of virtual prototype before and after correction

$\omega \quad$ angular velocity of driving wheel $\left(\mathrm{rad} \mathrm{s}^{-1}\right)$

$\omega_{z} \quad$ angular velocity of center-point steering motion without considering slippage $\left(\mathrm{rad} \mathrm{s}^{-1}\right)$

$\omega_{z r} \quad$ average value of center-point steering angular velocity of physical prototype in experiment $\left(\mathrm{rad} \mathrm{s}^{-1}\right)$

$\omega_{z s} \quad$ average value of center-point steering angular velocity of virtual prototype in simulation $\left(\mathrm{rad} \mathrm{s}^{-1}\right)$

$\omega_{z t} \quad$ angular velocity of center-point steering motion considering slippage $\left(\mathrm{rad} \mathrm{s}^{-1}\right)$

\section{Subscripts}

$i \quad$ different tracks on same tracked omni-vehicle, $i=1,2,3, \ldots n$

$k \quad$ different roller grounding points on same track, $k=0,1,2,3$

$p \quad$ different types of tracked omni-vehicle, $p=1,2,3$ 
Data availability. All the data used in this paper can be obtained from the corresponding author on request.

Author contributions. Conceptualization was by YF and YZ. Data curation was done by YF, TH, and MY. Formal analysis was made by YF. Investigations were carried out by YF, TH, and MY. The methodology was by YF and YZ. Project administration was performed by YZ and YS. Resources came from YZ. Software came from YF. Supervision was by YZ and YS. Visualization was by YF, TH, and MY. YF wrote the original draft. Writing, review, and editing were done by $\mathrm{YZ}$ and $\mathrm{YS}$.

Competing interests. The authors declare that they have no conflict of interest.

Acknowledgements. We thank LetPub (https://www.letpub.com, last access: 1 April 2021) for its linguistic assistance during the preparation of the manuscript.

Financial support. This research has been supported by the State Administration for Science, Technology and Industry for National Defense (grant no. 2015ZB15).

Review statement. This paper was edited by Daniel Condurache and reviewed by three anonymous referees.

\section{References}

Cao, A.: Omni-directional all-terrain six-wheeled tracked vehicle, Patent: National Intellectual Property Administration, PRC, CN106240663B, Beijing, China, 2018.

Chen, P., Mitsutake, S., Isoda, T., and Shi, T.: Omnidirectional robot and adaptive control method for offroad running, IEEE Trans. Robot. Autom., 18, 251-256, https://doi.org/10.1109/TRA.2002.999654, 2002.

Cheng, J., Gao, L., and Wang, H.: Steering analysis of tracked vehicles based on skid condition, Chinese J. Mech. Eng., 42, 192195, 2006.

Cheng, J., Gao, L., Wang, H., and Liu, F.: Analysis on the Steering of Tracked Vehicles, Acta Armamentarii, 28, 1110-1115, https://doi.org/10.3321/j.issn:1000-1093.2007.09.017, 2007.

Clavien, L., Lauria, M., and Michaud, F.: Instantaneous centre of rotation based motion control for omnidirectional mobile robots with sidewards off-centred wheels, Rob. Auton. Syst., 106, 5868, https://doi.org/10.1016/j.robot.2018.03.014, 2018.

Fang, Y., Zhang, Y., Li, N., and Shang, Y.: Research on a medium-tracked omni-vehicle, Mech. Sci., 11, 137-152, https://doi.org/10.5194/ms-11-137-2020, 2020.

Guan, J. and Yuan, K.: Omni-directional moving transmission continuous track, Patent: National Intellectual Property Administration, PRC, CN106515886B, Beijing, China, 2019.
Hua, J. and Zhang, C.: Four-axis drive crawler wheel type forklift, Patent: National Intellectual Property Administration, PRC, CN110356483A, Beijing, China, 2019.

Huang, T., Zhang, Y., Tian, P., Yan, N., and Zhang, J.: Design \& kinematics analysis of a tracked omnidirectional mobile platform, J. Mech. Eng., 50, 206-212, https://doi.org/10.3901/JME.2014.21.206, 2014.

Karavaev, Y. L. and Kilin, A. A.: Nonholonomic dynamics and control of a spherical robot with an internal omniwheel platform: Theory and experiments, Proc. Steklov Inst. Math., 295, 158167, https://doi.org/10.1134/s0081543816080095, 2017.

Li, X., Xie, F., Liu, D., Huang, L., Ye, Q., and Yuan, K.: Omnidirectional moving track, Patent: National Intellectual Property Administration, PRC, CN102756764A, Beijing, China, 2012.

Liu, Y., Huang, T., Li, H., and Yan, Y.: Combined crawler belt walking mechanism with omni-directional movement and platform thereof, Patent: National Intellectual Property Administration, PRC, CN108163072A, Beijing, China, 2018.

Ma, D., Chen, D., Li, M., Zhou, X., and Chen, Z.: Qxcomm technology's track based on mecanum wheel, Patent: National Intellectual Property Administration, PRC, CN204821767U, Beijing, China, 2015.

Madhushani, T. W. U., Maithripala, D. H. S., Wijayakulasooriya, J. V., and Berg, J. M.: Semi-globally exponential trajectory tracking for a class of spherical robots, Automatica, 85, 327-338, https://doi.org/10.1016/j.automatica.2017.07.060, 2017.

Mortensen Ernits, R., Hoppe, N., Kuznetsov, I., Uriarte, C., and Freitag, M.: A new omnidirectional track drive system for off-road vehicles, 2017 XXII Int. Conf., MATERIAL Handl. Constr. Logist., 105-110, 2017.

Park, Y. K., Lee, P., Choi, J. K., and Byun, K. S.: Analysis of factors related to vertical vibration of continuous alternate wheels for omnidirectional mobile robots, Intell. Serv. Robot., 9, 207-216, https://doi.org/10.1007/s11370-016-0196-3, 2016.

Roh, S.-G., Taguchi, Y., Nishida, Y., Yamaguchi, R., and Hirose, S.: Development of the portable ground motion simulator of an earthquake, in: 2013 IEEE/RSJ International Conference on Intelligent Robots and Systems (IROS), 5339-5344, https://doi.org/10.1109/IROS.2013.6697129, 2013.

Singh, A., Sachdeva, E., Sarkar, A., and Krishna, K. M.: COCrIP: Compliant omniCrawler in-pipeline robot, in: 2017 IEEE/RSJ International Conference on Intelligent Robots and Systems (IROS), IEEE, 5587-5593, https://doi.org/10.1109/IROS.2017.8206446, 2017.

Song, H., Gao, L., Li, J., and Yao, X.: Correction and Experiment of Steering Performance Index of Tracked Vehicles, J. Acad. Armored Force Eng., 22, 65-68, https://doi.org/10.3969/j.issn.1672-1497.2008.06.016, 2008.

Song, H., Yao, X., Gao, L., and Yu, K.: Correction and experiment of zero radio turning index of tracked vehicle, Veh. Power Technol., 3, 5-8, 17, https://doi.org/10.3969/j.issn.10094687.2009.03.002, 2009.

Tadakuma, K., Tadakuma, R., and Berengueres, J.: Tetrahedral Mobile Robot with Spherical Omnidirectional Wheel, J. Robot. Mechatronics, 20, 125-134, https://doi.org/10.20965/jrm.2008.p0125, 2008.

Tadakuma, K., Tadakuma, R., Nagatani, K., Yoshida, K., Aigo, M., and Shimojo, M.: Throwable tetrahedral robot with transforma- 
tion capability, 2009 IEEE/RSJ Int. Conf. Intell. Robot. Syst., 2801-2808, https://doi.org/10.1109/IROS.2009.5354196, 2009.

Tadakuma, K., Tadakuma, R., Higashimori, M., and Kaneko, M.: Robotic finger mechanism equipped omnidirectional driving roller with two active rotational axes, in: 2012 IEEE International Conference on Robotics and Automation, 3523-3524, https://doi.org/10.1109/ICRA.2012.6225378, 2012.

Tadakuma, K., Ogata, H., Higashimori, M., Kaneko, M., Tadakuma, R., and Berengueres, J.: Torus omnidirectional driving unit mechanism realized by curved crawler belts, Proc.-IEEE Int. Conf. Robot. Autom., 2567, https://doi.org/10.1109/ICRA.2014.6907224, 2014.

Tadakuma, K., Takane, E., Fujita, M., Nomura, A., Komatsu, H., Konyo, M., and Tadokoro, S.: Planar omnidirectional crawler mobile mechanism - development of actual mechanical prototype and basic experiments, IEEE Robot. Autom. Lett., 3, 395402, https://doi.org/10.1109/LRA.2017.2739101, 2017.

Takane, E., Tadakuma, K., Shimizu, T., Hayashi, S., Watanabe, M., Kagami, S., Nagatani, K., Konyo, M., and Tadokoro, S.: Basic Performance of Planar Omnidirectional Crawler during Direction Switching using Disturbance Degree of Ground Evaluation Method, IEEE Int. Conf. Intell. Robot. Syst., 2732-2739, https://doi.org/10.1109/IROS40897.2019.8968507, 2019.

Tong, Z.: A motion study of the omnidirectional mobile robot, Master Degree, Shenyang Aerospace University, Shen Yang, Liao Ning, China, 2017.

Wang, X.: Theory and application of Mecanum wheel based omnidirectional mobile, Southeast University Press, Jiang Su, 2018.

Xing, Q., Wu, H., Liu, Y., Yang, C., Zhu, Y., Liao, H., Cheng, S., and Wu, J.: Novel Mecanum wheel creeper truck, Patent: National Intellectual Property Administration, PRC, CN108016518A, Beijing, China, 2018.

Yang, H., Zhang, Y., Fang, Y., Cui, Z., and Dong, Z.: Analysis and Simulation of Omnidirectional Platform in Complex Environments, Fire Control Command Control, 44, 135-138, https://doi.org/10.3969/j.issn.1002-0640.2019.09.026, 2019a.

Yang, H., Zhang, Y., Huang, T., Fang, Y., Zhang, Q., and Zhang, J.: Motion analysis of novel track omnidirectional platform, J. Zhejiang Univ. Sci., 52, 1345-1353, https://doi.org/10.3785/j.issn.1008-973X.2018.07.015, 2019b.
Yang, Y., Yang, G., Tian, Y., Zheng, T., Li, L., and Wang, Z.: A robust and accurate SLAM algorithm for omnidirectional mobile robots based on a novel 2.5D lidar device, 2018 13th IEEE Conf. Ind. Electron. Appl., 2123-2127, https://doi.org/10.1109/ICIEA.2018.8398060, 2018.

Yu, C., Lin, J., and Liu, B.: Crawler-type omnidirectional mobile platform, Patent: National Intellectual Property Administration, PRC, CN210310616U, Beijing, China, 2020.

Zhang, J.: Omni-directional tracked vehicle, Patent: National Intellectual Property Administration, PRC, CN108583704A, Beijing, China, 2018.

Zhang, X., Yang, S., Sun, L., and Zhang, J.: Novel all-round track moving platform structure, Patent: National Intellectual Property Administration, PRC, CN205769664U, Beijing, China, 2016.

Zhang, Y.: Omnidirectional mobile track, Patent: National Intellectual Property Administration, PRC, CN103043128B, Beijing, China, 2017.

Zhang, Y. and Huang, T.: Research on a tracked omnidirectional and cross-country vehicle, Mech. Mach. Theory, 87, 18-44, https://doi.org/10.1016/j.mechmachtheory.2014.12.016, 2015.

Zhang, Y. and Yang, H.: Designing of a small reconnaissance platform based on omnidirectional chassis, Fire Control Command Control, 43, 130-134, https://doi.org/10.3969/j.issn.10020640.2018.07.024, 2018

Zhang, Y., Huang, T., Zhang, S., and Zhang, J.: Analysis about steering slip power ratio of a tracked omnidirectional mobile platform, Acta Armamentarii, 36, 1562-1568, https://doi.org/10.3969/j.issn.1000-1093.2015.08.026, 2015.

Zhang, Y., Yang, H., Huang, T., Zhang, S., and Fang, Y.: Analysis about motion of centripetal tracked omnidirectional mobile platforms, Acta Armamentarii, 38, 2309-2320, https://doi.org/10.3969/j.issn.1000-1093.2017.12.003, 2017.

Zhang, Y., Fang, Y., Yang, H., Cui, Z., and Shi, H.: Kinematics Analysis \& Simulation of a Tracked Omnidirectional Mobile Platform, Fire Control Command Control, 44, 132-136, https://doi.org/10.3969/j.issn.1002-0640.2019.06.026, 2019. 